Check for updates

Cite this: J. Mater. Chem. A, 2021, 9, 1192

Received 10th July 2020

Accepted 13th December 2020

DOI: $10.1039 / d 0 t a 06756 c$

rsc.li/materials-a

\section{Laser fabrication of hybrid electrodes composed of nanocarbons mixed with cerium and manganese oxides for supercapacitive energy storage $\dagger$}

\author{
Pablo García Lebière, (D) *a Ángel Pérez del Pino, (D) a Guillem Domènech Domingo, ${ }^{a}$ \\ Constantin Logofatu, (iD bd Immaculada Martínez-Rovira, (iD) ${ }^{c}$ Ibraheem Yousef (iDc ${ }^{c}$ \\ and Enikö György (DD) abd
}

\begin{abstract}
Novel composite materials are being investigated for improving the energy storage performance of electrochemical capacitors. For this goal, synergistic effects via the combination of diverse types of materials are crucial. In this work, electrodes composed of reduced graphene oxide, multiwall carbon nanotubes, as well as cerium and manganese oxides were fabricated through reactive inverse matrixassisted pulsed laser evaporation (RIMAPLE). UV-pulsed laser irradiation of frozen aqueous dispersions containing graphene oxide sheets, carbon nanotubes, and ceria nanoentities, besides manganese acetate precursor, led to the simultaneous chemical transformation and co-deposition of hybrid electrodes onto flexible metallic substrates via photothermal and photochemical processes. Thorough morphological and compositional studies of the electrodes demonstrated the laser-induced reduction of graphene oxide, besides the crystallization of a mixture of cerium and manganese oxide nanostructures decorating the carbon nanoentities during the deposition process. Electrochemical analyses revealed a remarkable improvement of performance with the combination of electrochemical double layer in the porous nanocarbon framework with pseudocapacitance from the oxide nanostructures, obtaining excellent volumetric capacitances of up to $140 \mathrm{~F} \mathrm{~cm}^{-3}$ at $10 \mathrm{mV} \mathrm{s}^{-1}$ with the combination of all four materials. The attained results are the best ones yet published regarding RIMAPLE of hybrid nanocarbon-based electrodes with micrometric thickness. Finally, symmetric electrochemical capacitors were fabricated using aqueous electrolyte, revealing excellent stability upon tens of thousands of charge-discharge cycles.
\end{abstract}

\section{Introduction}

The technological advance of portable consumer electronics, wearable medical devices, electric vehicles, smart electric grids and the emerging Internet of Things requires innovation in energy storage technologies. ${ }^{1}$ Without doubts, electrochemical energy storage technologies play a major role in this subject. In particular, supercapacitors are important for their fast and reversible use of energy, high power density, long cycle life, low maintenance cost, and environment-friendly nature. ${ }^{2,3}$ The storage mechanism of supercapacitors consists in two contributions: (i) the electrostatic accumulation of ionic charges from the electrolyte on the electrode's surface (electric double layer,

${ }^{a}$ Institute of Materials Science of Barcelona, ICMAB-CSIC, Campus UAB, 08193 Bellaterra, Spain. E-mail: pgarcia2@icmab.es; Tel: +34 935801853

${ }^{b}$ National Institute for Materials Physics, PO Box MG 7, 77125 Bucharest, Romania ${ }^{c} A L B A$ Synchrotron, Carrer de la Llum, 2-26, 08290 Cerdanyola del Vallès, Spain ${ }^{d}$ National Institute for Lasers, Plasma and Radiation Physics, PO Box MG 36, 77125 Bucharest, Romania

$\dagger$ Electronic supplementary information (ESI) available. See DOI: $10.1039 /$ dota06756c
EDL); and (ii) through surface reversible faradaic redox reactions at the surface of the active materials (pseudocapacitance). ${ }^{4}$ Carbon-based materials are widely investigated for supercapacitor electrodes using EDL mechanism (i.e. activated carbon, reduced graphene oxide, rGO; carbon nanotubes, $\mathrm{CNT}$ ). ${ }^{5-8}$ Many transition metal oxides (TMO) have been reported as either intrinsic or extrinsic pseudocapacitive materials, i.e. $\mathrm{RuO}_{2}, \mathrm{MnO}_{2}, \mathrm{Fe}_{3} \mathrm{O}_{4}$, NiO or $\mathrm{V}_{2} \mathrm{O}_{5}{ }^{4,8,9}$ Combining materials with both energy storage mechanisms can lead to synergistic effects, in which the electrochemical performance is enhanced, avoiding the low conductivity, poor stability and durability during charge/discharge processes common in metal oxides and preserving the advantages of rGO and CNT like high thermal conductivity, high electron mobility, mechanical stability and large surface area. ${ }^{\mathbf{8}, 10}$ Cerium dioxide $\left(\mathrm{CeO}_{2}\right)$ is an important rare earth metal oxide, abundant and inexpensive, which outstanding supercapacitive performance has been recently reported as nanostructures, ${ }^{11-13}$ in combination with carbon-based materials ${ }^{14,15}$ or other TMO. ${ }^{16,17}$ Despite its good redox characteristics, it suffers from poor conductivity and structure stability as stated before. Consequently, it is worth the study of this material in combination with carbon materials and 
TMO. In recent years, it has been proven that the electrical conductivity can be enhanced with the combination of two or more metal oxide materials. Hence, increasing charge transport improves their charge storage performance. ${ }^{18}$ These combinations of metal oxides possess different oxidation states enabling multiple redox reactions, enhancing rapid faradaic reactions from the metal species with synergistic effects. ${ }^{19}$ Transition metal oxides based on manganese $\left(\mathrm{MnO}_{x}\right)$ are promising materials with good electrochemical performance, low price, abundance, environmentally friendly and structural diversity. Although $\mathrm{MnO}_{2}$ is the most performant of the oxides, ${ }^{20} \mathrm{MnO}$ and $\mathrm{Mn}_{3} \mathrm{O}_{4}$ are also used for supercapacitor electrodes with good performance. ${ }^{21-24}$ Recently, many studies were focused on the study of supercapacitive behaviour of mixtures composed of two or more metal oxides. Regarding $\mathrm{CeO}_{2}$, enhanced performance has been reported in combination with cobalt ${ }^{\mathbf{1 6}}$ and $\mathrm{ZnO},{ }^{25}$ and for manganese oxides, in combination with $\mathrm{Co}_{3} \mathrm{O}_{4}{ }^{26}$ $\mathrm{ZnO},{ }^{27} \mathrm{Fe}_{2} \mathrm{O}_{3},{ }^{28} \mathrm{Cu}_{2} \mathrm{O},{ }^{29}$ and different metal ions as cobalt, copper, nickel or chromium. ${ }^{30,31}$ Moreover, many reports combined $\mathrm{MnO}_{2}$ and $\mathrm{CeO}_{2}$ with outstanding performance ${ }^{32-36}$ but a limited number of reports deal with $\mathrm{Mn}_{3} \mathrm{O}_{4} \cdot{ }^{37}$ In summary, the synergistic effect obtained from the combination of cerium and manganese oxide materials might point to a promising composite for supercapacitive devices.

The synthesis of carbon-based materials decorated with metal-oxide nanostructures can be achieved by laser-based techniques. These fabrication methods have a high potential for fast and versatile production of flexible supercapacitor electrodes and devices avoiding lasting high-temperature conditions and toxic chemicals. ${ }^{38,39}$ Importantly, laser methods produce very fast photolysis and heating of the irradiated material leading to the development of chemical reactions and phase changes out of the thermodynamic equilibrium that cannot be developed through conventional techniques. Additionally, laser heating can change the thermo-optical properties of the medium introducing non-linearities in the interaction process. Therefore, unusual chemical pathways and reaction products, novel material microstructures and phases, and new morphologies can be obtained. ${ }^{40}$ Considering these advantages, reactive inverse matrix-assisted pulsed laser evaporation (RIMAPLE) stands up as a promising method for the fabrication of composite films formed by carbon nanomaterials coated with transition metal oxide nanostructures for supercapacitor electrodes. ${ }^{41-46}$ In this approach, the coating of the carbon nanomaterials is provided by the melting and recrystallization of TMO nanoparticles (NPs) present in the target. Remarkably, Steiner et al. proved that MAPLE technique also allows the deposition of metal oxides nanoparticles using aqueous solutions of metal organic precursors (acetates) in the frozen targets. ${ }^{47}$ Thus, the UV photon energy and developed temperature rise are high enough for the laser-induced decomposition of the acetate precursors, allowing the free metal ions to crystallize nanostructures within the target before the ejection of material. To the best of our knowledge, the combined deposition of nanoentities by using metal organic precursors has not been addressed so far.
In this work, we present a novel RIMAPLE method for the synthesis of micrometric-thick hybrid electrodes composed of rGO and multiwall carbon nanotubes (MWCNTs) decorated with cerium and manganese oxides by combination of $\mathrm{CeO}_{2}$ NPs and Mn-based metal organic precursors in the target. Laserinduced photothermal/photochemical mechanisms led to the formation of $\mathrm{CeO}_{2}$ and $\mathrm{Ce}_{2} \mathrm{O}_{3}$ besides $\mathrm{MnO}$ and $\mathrm{Mn}_{3} \mathrm{O}_{4}$ nanostructures on the surface of rGO sheets and MWCNTs. The morphological, structural and electrochemical properties of the obtained electrodes were investigated. The contribution of the different components to the electrochemical performance was also studied. It is noted a significant increase of the volumetric capacitance in the final composite due to the insertion of CNTs that prevents re-stacking of rGO sheets and increases the porosity, ${ }^{48}$ as well as the increase of pseudocapacitance provided by cerium and manganese oxides. As a proof of concept a symmetric electrochemical capacitor was assembled to test the fabricated electrodes in practical use, exhibiting outstanding stability performance upon cycling.

\section{Experimental}

\subsection{Samples fabrication}

RIMAPLE targets were prepared by mixing different aqueous dispersions composed of graphene oxide (GO) powder (NanoInnova Technologies), multiwall carbon nanotubes (SigmaAldrich), cerium(Iv) oxide nanoparticles (Sigma-Aldrich) and manganese(II) acetate (Sigma-Aldrich). GO powder was composed of 1-15 layers thick flakes around $1 \mu \mathrm{m}^{2}$ in lateral size. Carbon nanotubes were about $10 \mathrm{~nm}$ in diameter and up to $1.5 \mu \mathrm{m}$ in length, doped with carboxylic groups for enhancing their dispersibility. $\mathrm{CeO}_{2}$ nanoparticles were about $25 \mathrm{~nm}$ in diameter. The concentration of $\mathrm{GO}$ and $\mathrm{CeO}_{2}$ nanoparticles were chosen as $5 \mathrm{wt} \%$ for preventing precipitation and excessively high viscosity of the dispersions, and for obtaining high rate deposition process. The concentration of the rest of the precursors was varied to investigate their influence in the electrodes' performance. Table 1 shows the relative concentration of the precursors present in the dispersions. Once synthesized, the dispersions were stirred and sonicated at room temperature. MAPLE targets were prepared by filling $3 \mathrm{~mL}$ aluminium holder and immediately flash frozen submerging it in liquid- $\mathrm{N}_{2}$. Finally, the holders with the frozen dispersions were placed inside the deposition chamber and remained frozen during the deposition process by using a liquid $\mathrm{N}_{2}$ cooled holder. This flash-freezing method ensures the homogeneity of the target dispersion and allows a congruent deposition of the material.

The films were deposited by accumulation of $6000 \mathrm{UV}$ laser pulses (266 nm wavelength, $\approx 4 \mathrm{~ns}$ pulse duration, $10 \mathrm{~Hz}$ repetition rate) with $0.4 \mathrm{~J} \mathrm{~cm}^{-2}$ laser fluence submitted to the frozen target using a Quantel Brilliant B Nd:YAG laser system. During the deposition, the laser beam scanned the target surface at an incident angle of $45^{\circ}$. Flexible and conducting $1 \times$ $1 \mathrm{~cm}^{2}$ substrates made of (i) $0.5 \mathrm{~mm}$-thick copper and (ii) 0.1 mm-thick stainless steel AISI 316L, were positioned in front of the target at a separation distance of $4 \mathrm{~cm}$. The deposition 
Table 1 Relative concentration of precursors in the aqueous dispersions for MAPLE targets

\begin{tabular}{|c|c|}
\hline Dispersion & Composition \\
\hline GO-Ce & $\mathrm{GO}(5 \mathrm{wt} \%)+\mathrm{CeO}_{2} \mathrm{NPs}(5 \mathrm{wt} \%)$ \\
\hline CNT-Ce & MWCNT $(2 w t \%)+\mathrm{CeO}_{2}$ NPs $(5 \mathrm{wt} \%)$ \\
\hline GO-CNT-Ce-525 & $\mathrm{GO}(5 \mathrm{wt} \%)+\mathrm{MWCNT}(2 \mathrm{wt} \%)+\mathrm{CeO}_{2} \mathrm{NPs}(5 \mathrm{wt} \%)$ \\
\hline GO-CNT-Ce-Mn-5151 & $\mathrm{GO}(5 \mathrm{wt} \%)+\mathrm{MWCNT}(1 \mathrm{wt} \%)+\mathrm{CeO}_{2} \mathrm{NPs}(5 \mathrm{wt} \%)+\mathrm{Mn}\left(\mathrm{CH}_{3} \mathrm{CO}_{2}\right)_{2}(1 \mathrm{wt} \%)$ \\
\hline GO-CNT-Ce-Mn-5152 & $\mathrm{GO}(5 w \mathrm{t} \%)+\mathrm{MWCNT}(1 \mathrm{wt} \%)+\mathrm{CeO}_{2} \mathrm{NPs}(5 \mathrm{wt} \%)+\mathrm{Mn}\left(\mathrm{CH}_{3} \mathrm{CO}_{2}\right)_{2}(2 \mathrm{wt} \%)$ \\
\hline
\end{tabular}

process was performed at room temperature, in a $20 \mathrm{~Pa} \mathrm{~N}_{2}$ background gas environment after evacuation to a residual pressure of $0.1 \mathrm{~Pa}$.

A symmetric electrochemical capacitor was assembled with the best performant electrode by using stainless steel AISI 316L substrate $\left(1 \mathrm{~cm}^{2}\right.$ area, $0.1 \mathrm{~mm}$ thickness) as current collector. The electrodes were separated by glass microfiber filter paper (Prat Dumas) impregnated with $1 \mathrm{M} \mathrm{Na}_{2} \mathrm{SO}_{4}$ aqueous electrolyte. A few cm long copper contacts were attached to the steel current collector and the system was sealed with plastic film using an impulse heat sealer type IS300C.

\subsection{Characterization techniques}

Thermal simulations of the GO, CNT and $\mathrm{CeO}_{2}$ nanoparticles within the water ice matrix were performed by solving the transient heat equation using the finite element method with COMSOL 5.5 software. The thickness and the morphology of the composite films were studied by field emission scanning electron microscopy (SEM) using QUANTA 200 FEG-ESEM and extreme high resolution (XHRSEM) Magellan $400 \mathrm{~L}$ microscopes (FEI). The study of their structural properties was performed through high resolution transmission electron microscopy (HRTEM) and high-angle annular dark field (HAADF) imaging in high resolution scanning transmission electron microscopy (STEM) through a Tecnai F20 equipment (FEI). The chemical composition of the fabricated films was investigated by X-ray photoelectron spectroscopy (XPS) using a SPECS XPS spectrometer based on a Phoibos 150 electron energy analyser operated in a constant energy mode. The system used the aluminium anode (Al $\mathrm{K} \alpha 1486.74 \mathrm{eV})$ as a monochromatic X-ray source. Wide range spectra over $1500 \mathrm{eV}$ binding energies were recorded using a $50 \mathrm{eV}$ analyser pass energy. High resolution spectra were also acquired over $20 \mathrm{eV}$ ranges at $10 \mathrm{eV}$ pass energy with an energy resolution of $0.7 \mathrm{eV}$. All the XPS measurements were performed in ultra-high vacuum $\left(\approx 10^{-7} \mathrm{~Pa}\right)$. Synchrotron-based Fourier Transform Infrared Microspectroscopy (SR-FTIRM) was also used to study the chemical composition of the composite materials. This analysis was carried out at the infrared beamline MIRAS of ALBA synchrotron light source using a Hyperion 3000 microscope coupled to a Vertex 70 spectrometer (Bruker, Germany). The microscope is equipped with a liquid $\mathrm{N}_{2}$-cooled mercury cadmium telluride $50 \mu \mathrm{m}$ MCT detector. A 36× Schwarzschild objective (NA $=0.52$ ) was used and all the spectra were collected in the trans-reflection mode using a single masking aperture size of $10 \times 10 \mu \mathrm{m}^{2}$. A collection of 9 spectra for each sample was obtained in the $4000-600 \mathrm{~cm}^{-1}$ mid-infrared spectral range at $4 \mathrm{~cm}^{-1}$ spectral resolution with 1024 co-added scans per spectrum. For the correct identification of bands, baselines were subtracted.

The electrochemical performance of the fabricated electrodes was investigated through cyclic voltammetry (CV) in the voltage range [0, 0.8] V using a Keithley 2450-EC Electrochemistry Lab System. The samples deposited on stainless steel were used as working electrodes in a three-electrode configuration. The analyses were performed in a $0.5 \mathrm{~cm}^{2}$ samples area using a plate material electrochemical cell (Bio-logic), and $\mathrm{Ag} / \mathrm{AgCl}$ reference electrode (3 $\mathrm{M} \mathrm{KCl}$ internal solution), a $\mathrm{Pt}$ wire counter electrode and $1 \mathrm{M} \mathrm{Na}_{2} \mathrm{SO}_{4}$ aqueous solution as electrolyte at room temperature. Galvanostatic charge-discharge (GCD) analyses were performed with the same cell configuration as CV studies changing the current density. Electrochemical impedance spectroscopy (EIS) measurements were also fulfilled through a Hioki IM3590 chemical impedance analyser. Impedance values were acquired in the frequency range of $1 \mathrm{~Hz}$ to $200 \mathrm{kHz}$ at open circuit potential with an amplitude perturbation of $5 \mathrm{mV}$. An average of 5 measurements per data point was obtained to minimize the experimental error. Regression of EIS data to equivalent circuit models was performed using EIS Spectrum Analyser software.

\section{Results and discussion}

The targets submitted to laser radiation are composed of a mixture of GO sheets, MWCNTs and $\mathrm{CeO}_{2}$ NPs homogeneously dispersed in a frozen matrix of water at a measured temperature of about $-30{ }^{\circ} \mathrm{C}$. Due to the low temperature of the matrix, the targets having $\mathrm{Mn}$ precursor are considered to mainly contain aggregated $\mathrm{Mn}\left(\mathrm{CH}_{3} \mathrm{COO}\right)_{2}$ molecules. Fig. 1a displays a scheme of the proposed mechanisms occurring during laser irradiation of the target. Photochemical processes involve the direct breaking of chemical bonds and photothermal interactions are caused by the thermalization of the excited electrons having interaction times longer than photochemical processes ones. ${ }^{49}$ Preceding studies proved the photochemical reduction of GO solutions by UV light. ${ }^{50}$ Using the laser radiation with $266 \mathrm{~nm}$ wavelength, which corresponds to $c a .4 .7 \mathrm{eV}$ photon energy, there is enough energy for the reduction taking into account that the threshold was determined to be $3.2 \mathrm{eV}^{50}$ Therefore, the photochemical processes 

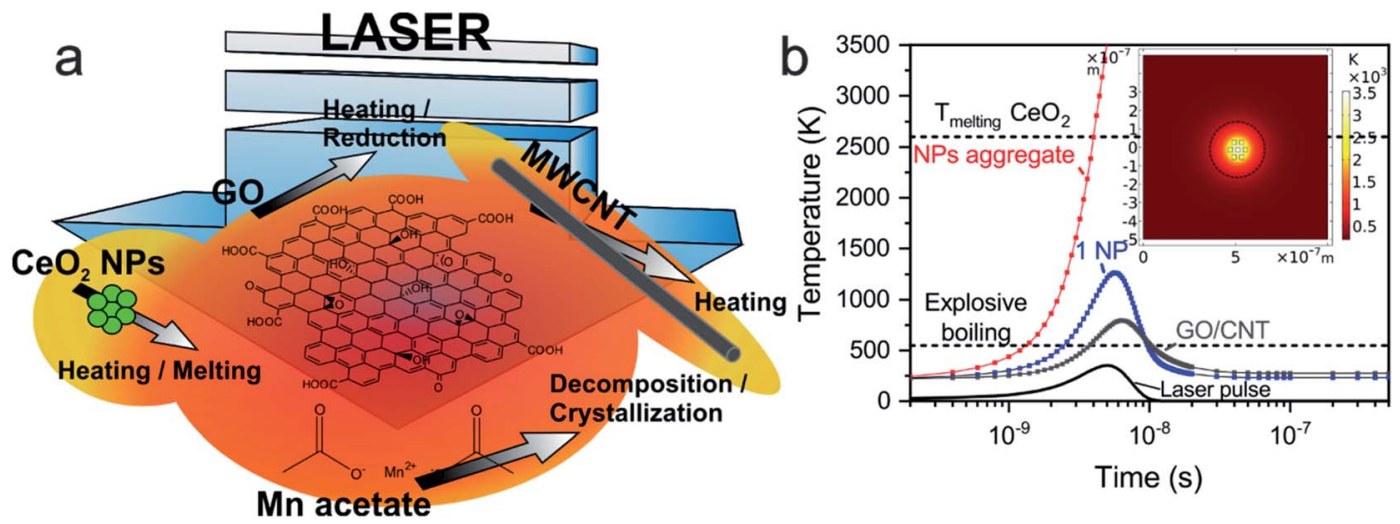

Fig. 1 (a) Scheme of the photothermal and photochemical mechanisms involved during laser irradiation, and (b) simulated temperature of $\mathrm{CeO}_{2}$ NPs and carbon-based materials during laser irradiation. Inset: temperature map of an aggregate of 6 NPs surrounding a single one. Circle indicates the surrounding region with enough temperature for manganese acetate decomposition.

are considered to be the dominating ones for GO reduction ${ }^{51}$ even though photothermal processes also take place. Regarding graphitic domains of GO and MWCNTs, the main process is considered to be photothermal due to the lack of enough photonic energy to break $\mathrm{C}=\mathrm{C} \mathrm{sp}{ }^{2}$ bonds $(6.4 \mathrm{eV}) .^{52}$ The UV radiation excites both $\sigma$ and $\pi$-bonded electrons constituent of graphene and CNT structures, leading to their fast heating. ${ }^{53}$ Considering the absorption of $\mathrm{CeO}_{2}$ nanoparticles (bandgap of ca. $3.2 \mathrm{eV}^{54}$ ), the excited electrons would also lead to significant heating by electron-phonon scattering. It is noteworthy that they would not be energetic enough for the $\mathrm{CeO}_{2}$ reduction to form $\mathrm{Ce}_{2} \mathrm{O}_{3}{ }^{55}$ despite that photochemical processes drive the exothermal reduction of GO producing a large amount of heat. ${ }^{56}$ Nevertheless, the presence of intrinsic oxygen vacancies from the nanoparticle lattice or laser-induced defects may generate a reversible transition between the cerium oxidation states $\mathrm{Ce}^{4+}$ and $\mathrm{Ce}^{3+} \cdot{ }^{57}$ Lastly, the laser radiation absorbed by manganese acetate molecules has enough energy to break the $\mathrm{Mn}-\mathrm{O}$ bond from the acetate (photodissociation process). ${ }^{55}$ However, Steiner et al. established that in reactive MAPLE process the decomposition of acetates is dominated by photothermal pathways and their deposition can be limited by a water explosive boiling temperature lower than the decomposition one ${ }^{47}$ In the present work, the main contribution of thermal energy required to reach the decomposition temperature of the $\mathrm{Mn}$ acetate is due to $\mathrm{GO}$, CNTs and $\mathrm{CeO}_{2}$ NPs, which rapidly heat the matrix surrounding them beyond the acetate decomposition temperature (over $600 \mathrm{~K}$ )..$^{58,59}$ Additionally, photochemical processes can occur producing diverse species of manganese oxide. ${ }^{60}$

Previous works studied UV laser irradiation of GO, MWCNT and different types NPs immersed in a water-ice target during RIMAPLE deposition process. ${ }^{61,62}$ In this work, $\mathrm{CeO}_{2}$ NPs were used for the first time in RIMAPLE experiments. Considering the thermophysical ${ }^{63}$ and optical properties ${ }^{64}$ of $\mathrm{CeO}_{2} \mathrm{NPs}$, the temperature evolution was simulated upon one laser pulse irradiation. As previously reported, water ice is transparent to the used UV radiation though the nanocarbon materials and the oxide NPs absorb it, being rapidly heated. As observed in
Fig. 1b, the sudden heat released by a single NP or a carbon nanoentity (GO, CNT) leads to an explosive boiling of the surrounding water ice resulting in the deposition of material onto the facing substrate. ${ }^{45,47}$ The laser-induced chemical reactions cause the reduction of GO, resulting in a material similar to pristine graphene, called reduced graphene oxide $(\mathrm{rGO})^{62}$ and the thermally-driven recrystallization of the metal oxide nanostructures, which rise over $1000{ }^{\circ} \mathrm{C}$ during several ns. The photothermal simulations indicate that the individual heating of a $\mathrm{CeO}_{2} \mathrm{NP}$ is far from reaching its melting temperature. Nonetheless, it is well known the tendency of NPs to aggregate. Laser irradiation of small NPs aggregates with at least double the radius of a single one is enough to induce the $\mathrm{CeO}_{2}$ phase change as it is demonstrated in Fig. 1b. Moreover, the reached matrix temperature in a radius of $c a .200 \mathrm{~nm}$ around $\mathrm{CeO}_{2} \mathrm{NPs}$, GO and CNTs would be enough for the decomposition of manganese acetate molecules, producing the crystallization of its oxides, as previously mentioned. Seven different samples were prepared (Table 1): four of them to optimize the concentration of GO-CNT-CeO ${ }_{2}$ and three with different concentration of manganese acetate. The electrodes will be referred onwards as their corresponding dispersion. The fabricated films are adhered to the flexible substrate and show homogeneous black colour. As seen in Table S1, $\dagger$ film thickness is similar in all of them, varying in the range of 1.1-1.7 $\mu \mathrm{m}$.

\subsection{Morphological characterization}

Morphological XHRSEM analyses reveal that all the fabricated samples present a homogeneous structure without visible differences at low magnification with the addition of the different compounds (Fig. S1 $\dagger$ ). At higher magnification, the homogeneity and evenly distribution of compounds of the GOCe (Fig. S2a $\dagger$ ) and CNT-Ce (Fig. S2b†) electrodes are clear. Both carbon-based materials present neither visible structural damage nor wrinkles. The combination of GO, CNT and $\mathrm{CeO}_{2}$ NPs (Fig. S2c $\dagger$ ), produces a similar structure than in the previous cases but with a visible variation of porosity. However, the addition of manganese acetate does not reflect significant 

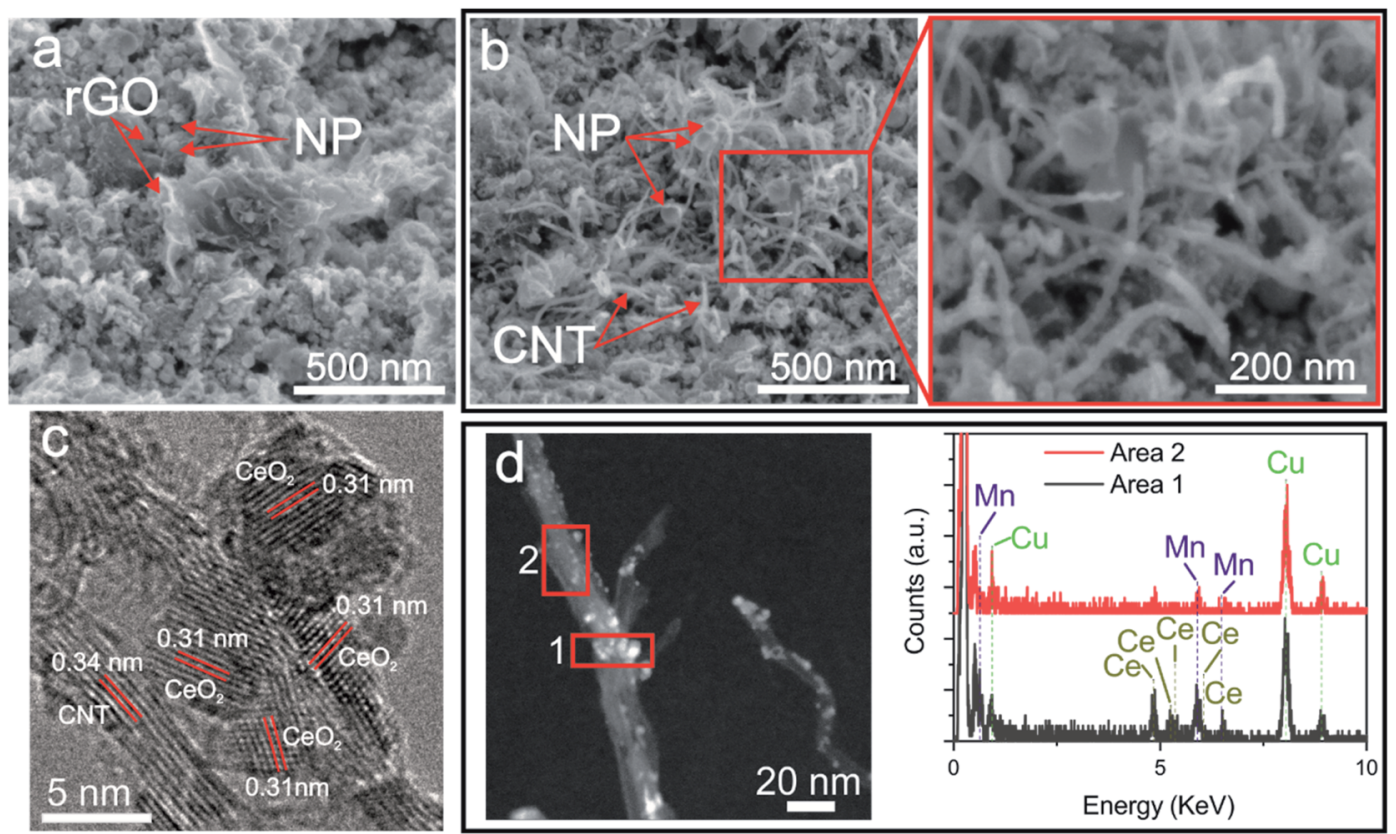

Fig. 2 XHRSEM images of (a) GO-Ce, and (b) GO-CNT-Ce-Mn-5152 with magnified region on the right; (c) HRTEM image of CNT-Ce; (d) HAADF-STEM image of a nanotube of GO-CNT-Ce-Mn-5152 with EDX spectra of the depicted regions.

differences. At even higher magnification, there is no obvious difference in the samples with the increasing amount of manganese acetate (Fig. S3†). Focusing on CNTs amount, Fig. S4† illustrates influence in morphology by the addition of a higher concentration. The addition of $2 \mathrm{wt} \%$ of CNTs causes their agglomeration (Fig. S4b†), not noticeable in lower concentration where the CNTs are evenly distributed. Fig. 2a and $b$ show various XHRSEM images where it can be differentiated the presence of rGO sheets and CNTs randomly distributed and decorated with nanostructures. Interestingly, it can also be witnessed the formation of nanostructures along the
CNTs (zoomed region of Fig. 2b). Focusing on the size of nanoparticles, average diameters in the range of 5-75 $\mathrm{nm}$ were measured, though highest resolution images demonstrate sizes as small as $1.5 \mathrm{~nm}$. This size range, different from that of raw $\mathrm{CeO}_{2} \mathrm{NPs}(25 \mathrm{~nm})$ is due to laser-induced structural-chemical transformation during the deposition process. Molten $\mathrm{CeO}_{2}$ NPs would suffer dewetting, forming smaller particles, besides coalescence, forming larger ones. In the samples obtained with targets containing $\mathrm{Mn}$ acetate, the formation of manganese oxide crystallites is expected from $\mathrm{Mn}$ ions dissolved in boiling water, generating nanostructures with different sizes in the
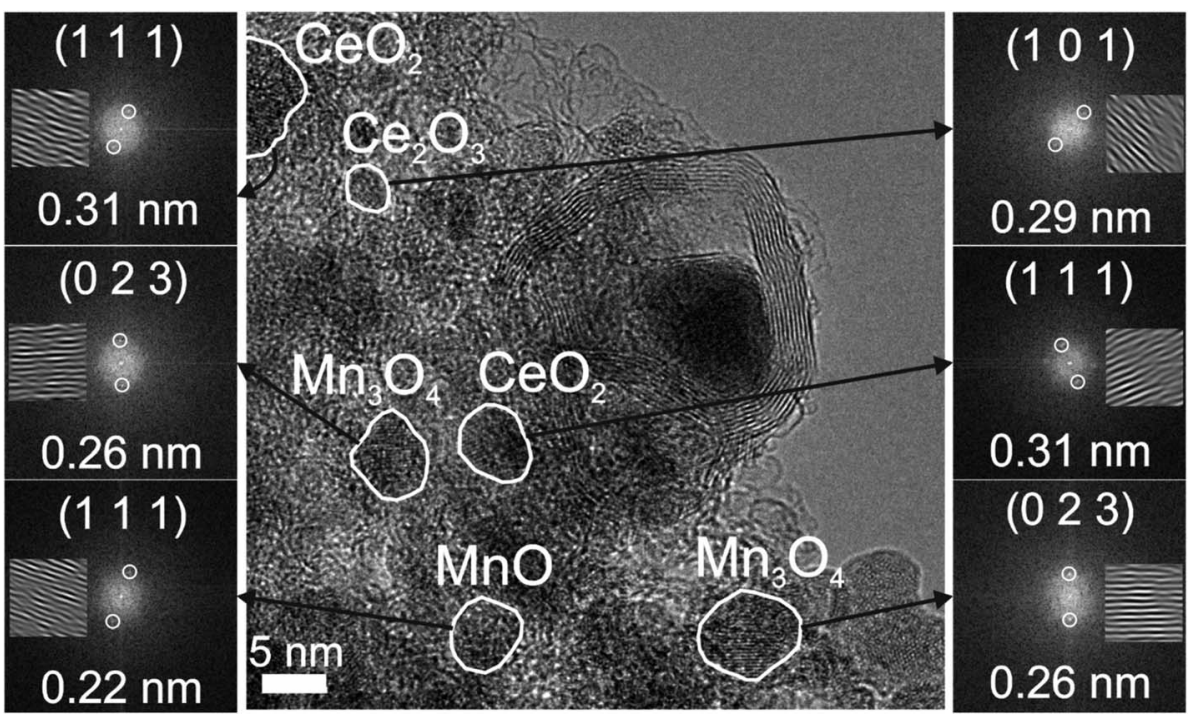

Fig. 3 HRTEM image of GO-CNT-Ce-Mn-5155 with FFT and filtrated lattices of the specified regions. 
fabricated electrode. In Fig. 2c, a CNT (0.34 nm interplanar distance) covered by nanocrystals is shown by HRTEM. The crystal structures observed on the surface show interplanar distance of $0.31 \mathrm{~nm}$ corresponding to (111) $\mathrm{CeO}_{2}$ (JCPDS 00-0040593). These structures, about $5 \mathrm{~nm}$ in size, are located at the surface of the CNTs and do not appear highly aggregated. It is worth noting that this crystal distribution is suitable for energy storage because the CNT works both as scaffold and as current collector for the insulating metal oxides, which store energy through pseudocapacitance. Moreover, the small size of the oxide crystals allows maintaining their electric resistance low. Additional HRTEM image of electrodes containing rGO, CNT and ceria NPs show different crystalline domains compatible with (101) $\mathrm{Ce}_{2} \mathrm{O}_{3}$ (JCPDs 00-023-1048) besides (111) $\mathrm{CeO}_{2}$, with varying size domains from 5 to $35 \mathrm{~nm}$ (Fig. S5†). The change in the oxidation state of Ce ions proves that ceria suffers reduction processes during deposition. The HRTEM image shown in Fig. 3 reveals different crystal structures identified in GO-CNT-Ce-Mn-
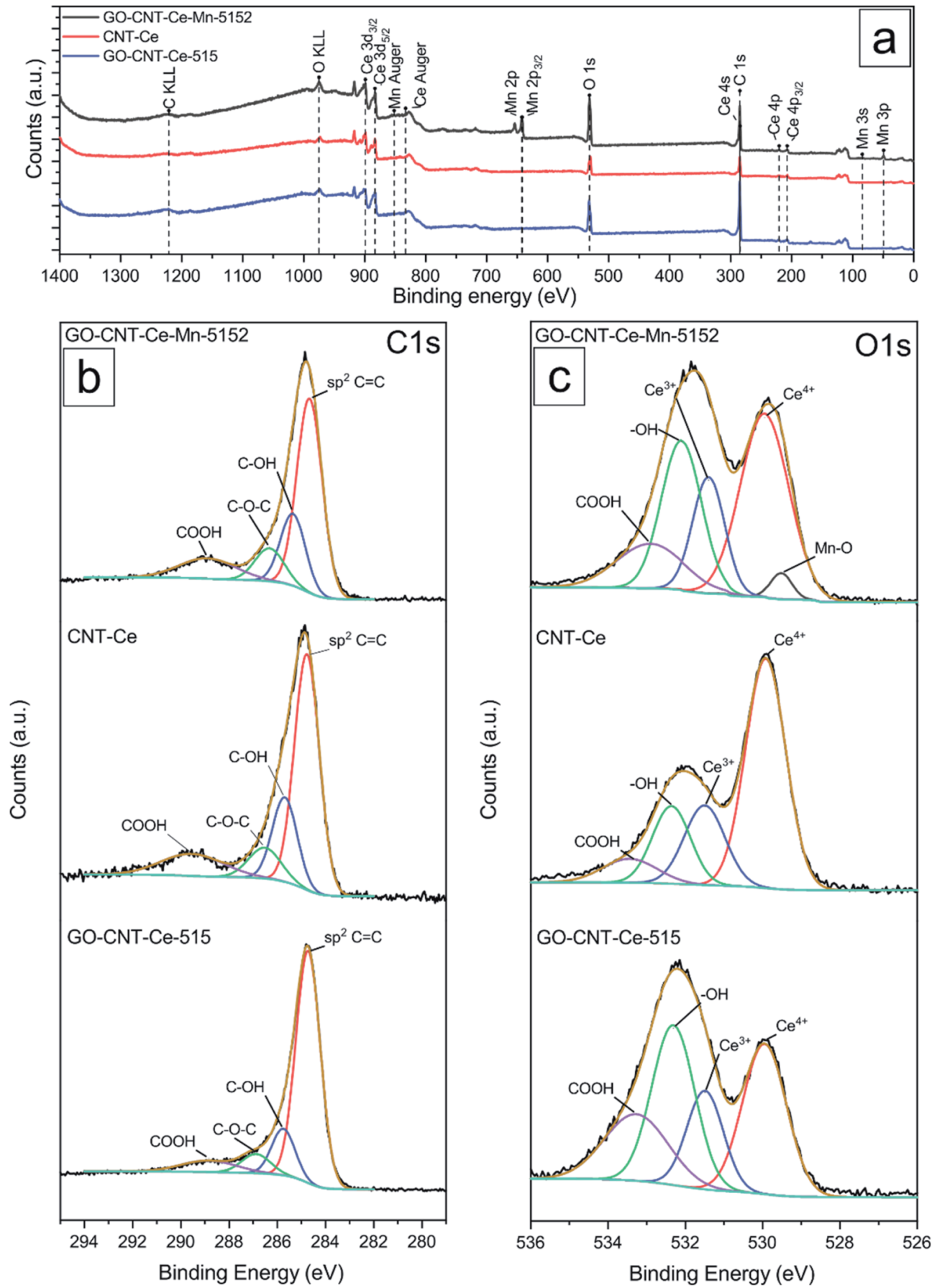

Fig. 4 (a) Wide XPS spectra and fitting curves of high-resolution (b) C 1s and (c) O 1s spectra of samples GO-CNT-Ce-Mn-5152, CNT-Ce and GO-CNT-Ce-515. 
5155, a sample where $\mathrm{Mn}$ component is present. Both $\mathrm{CeO}_{2}$ and $\mathrm{Ce}_{2} \mathrm{O}_{3}$ were observed, being the same crystal structures found in samples without Mn. At the same time, the Mn oxide species are $\mathrm{MnO}$ (JCPDS 04-005-4310) and $\mathrm{Mn}_{3} \mathrm{O}_{4}$ (JCPDS 01-075-0765). This corroborates the decomposition of the original acetate and the crystallization mechanism forming oxides. In this case, the Mn ions suffer oxidation during laser irradiation since $\mathrm{Mn}_{3} \mathrm{O}_{4}$ has two different states of oxidation $\left(\mathrm{Mn}^{2+}, \mathrm{Mn}^{3+}\right)$ that were not present in the original compound (only $\mathrm{Mn}^{2+}$ in the acetate). Both Ce- and Mn-based nanostructures can be found on rGO (Fig. 3) and CNT, as seen in HAADF-STEM analysis shown in Fig. 2d. Importantly, it can be determined that both $\mathrm{Mn}$ and Ce oxides can be found in the same CNT but without sharing position. Hence, there is no indication of the formation of mixed oxides during the deposition process. The average size of NPs in the Ce region (1) is $2.8 \mathrm{~nm}$ and in the Mn region (2) is $1.7 \mathrm{~nm}$, being slightly smaller Mn structures than Ce ones in general.

\subsection{Compositional characterization}

Compositional analyses of the fabricated electrodes were performed using XPS and SR-FTIRM. Fig. 4a shows a wide XPS spectrum identifying C, O, Ce and Mn XPS peaks besides some other Auger peaks of characteristic samples (CNT-Ce, GO-CNTCe-515 and GO-CNT-Ce-Mn-5152). All three spectra contain equivalent peaks at wide XPS, with the presence of Mn related peaks on the GO-CNT-Ce-Mn-5152 spectrum. No signal of nitrogen is recorded, revealing a negligible incorporation of $\mathrm{N}$ from background gas during the deposition process. In Fig. $4 \mathrm{~b}$ and $\mathrm{c}$ the $\mathrm{C} 1 \mathrm{~s}$ and $\mathrm{O} 1 \mathrm{~s}$ high-resolution spectra of characteristic electrodes are presented, respectively. $\mathrm{C} 1 \mathrm{~s}$ peak is deconvoluted into $\mathrm{sp}^{2}$-bonded carbon $(284.8 \mathrm{eV})$ probably containing some contribution of $\mathrm{sp}^{3}$ bonds, and carbon-oxygen hydroxyl (285.7 eV), epoxide (286.5 eV) and carboxylic (289.5 eV) groups. ${ }^{61}$ Note the absence of carbonyl groups, generally present in RIMAPLE samples containing $\mathrm{NiO}, \mathrm{TiO}_{2}$ and $\mathrm{Fe}_{3} \mathrm{O}_{4}$ NPs. ${ }^{41,61,65}$ Analysing the percentage of each peak, similar results are obtained between the three electrodes (Fig. S6a $\dagger$ ). Regarding the GO-CNT-Ce-515 sample, the $\mathrm{sp}^{2}$-bonded $\mathrm{C}=\mathrm{C}$ contribution is higher than in the others, indicating a greater graphitic structure restitution, even though this signal is the main contribution in all of them. Furthermore, O 1s signal is deconvoluted in $\mathrm{Ce}^{4+}(529.95 \mathrm{eV}), \mathrm{Ce}^{3+}(531.4 \mathrm{eV})$ also related to oxygen vacancies
$\mathrm{O}^{2-}$ in the lattice of metal oxide, hydroxyl (532.15 eV) and carboxyl $(532.91 \mathrm{eV})$ bond contributions. ${ }^{66-68}$ In the samples including manganese acetate in the dispersion, the deconvolutions also include the $\mathrm{O}^{-2}$ structural peak, related to $\mathrm{Mn}-\mathrm{O}-$ Mn bond (529.64 eV). ${ }^{69,70}$ This peak is the most intense in the $\mathrm{O}$ 1s spectra of Mn oxides ( $\mathrm{ca}$. 80\%). Therefore, the contribution of hydroxyl and water peaks is negligible. Using the $\mathrm{Ce}^{4+}$ and $\mathrm{Ce}^{3+}$ peaks, the relative content of each is calculated with the relative integrated area of the constituent peaks, revealing similar percentage of $\mathrm{Ce}^{4+}$ in CNT-Ce, GO-CNT-Ce-515 and GO-CNT-CeMn-5152 (73\%, 61\% and 71\%, respectively) (Fig. S6b $\dagger$ ). For a better enquiry of the study of Ce states, Fig. 5 shows the deconvolution of the Ce $3 \mathrm{~d}_{3 / 2}$ and $3 \mathrm{~d}_{5 / 2}$ signals. The six peaks labelled as $v_{0}, v_{1}, v_{2}\left(3 d_{5 / 2}\right)$ and $v_{0}^{\prime}, v_{1}^{\prime}$ and $v_{2}^{\prime}\left(3 d_{3 / 2}\right)$ refer to the three pairs of spin-orbit doublets characteristic of $\mathrm{Ce}^{4+} 3 \mathrm{~d}$ final states. Another two pairs of spin-orbit doublets $\mathrm{u}_{0} / \mathrm{u}_{0}^{\prime}$ and $\mathrm{u}_{1} / \mathrm{u}_{2}^{\prime}$ correspond to $\mathrm{Ce}^{3+} \cdot{ }^{71}$ It is worth noting that these doublets are not present in the XPS spectrum of raw-CeO nanoparticles, as seen in Fig. S7. $\dagger$ As for samples containing $\mathrm{Mn}$, the Ce $3 \mathrm{~d}_{3 / 2}$ line overlaps with the Auger Mn line LMM $(900 \mathrm{eV})$. Since in this case a reasonable deconvolution cannot be performed for the calculation of $\mathrm{Ce}^{4+}$, we use the relation

$$
\mathrm{Ce}^{4+0} \%=\frac{\mathrm{v}_{2}^{\prime} \%}{14} \times 100 \%
$$

where $v_{2}^{\prime} \%$ is the percentage of $v_{2}^{\prime}$ peak area respect to the total Ce $3 \mathrm{~d}$ area, with the relative error in the range of $10 \% .{ }^{72}$ Using this relation, the percentage of $\mathrm{Ce}^{4+}$ for raw- $\mathrm{CeO}_{2}$ nanoparticles is $100 \%$, for GO-CNT-Ce-Mn-5152 is $78 \%$, for CNT-Ce of $\mathrm{Ce}^{4+}$ is $69 \%$ and $70 \%$ for GO-CNT-Ce-515. The three last values are similar to the ones obtained from the study of $\mathrm{O} 1 \mathrm{~s}$ band. All three spectra from the fabricated electrodes present similar percentage of $\mathrm{Ce}^{3+}$ and $\mathrm{Ce}^{4+}$. The coexistence of both states leads to the presence of $\mathrm{Ce}_{2} \mathrm{O}_{3}$ and $\mathrm{CeO}_{2}$ as determined with HRTEM analyses. Moreover, there is a possibility of the existence of intermediate states of $\mathrm{CeO}_{2-x}$ with partially reduced $\mathrm{CeO}_{2}$. Therefore, some of the original NPs suffer reduction during the deposition process, changing from $\mathrm{Ce}^{4+}$ to $\mathrm{Ce}^{3+}$ state. Taking into account that stoichiometric $\mathrm{CeO}_{2}$ is an insulator, this reduction is important for enhancing the capability to store and mobilize oxygen within the ceria matrix and for increasing the electronic conduction attributed to the formation of small polarons which follow a hopping mechanism to diffuse. ${ }^{68,73}$

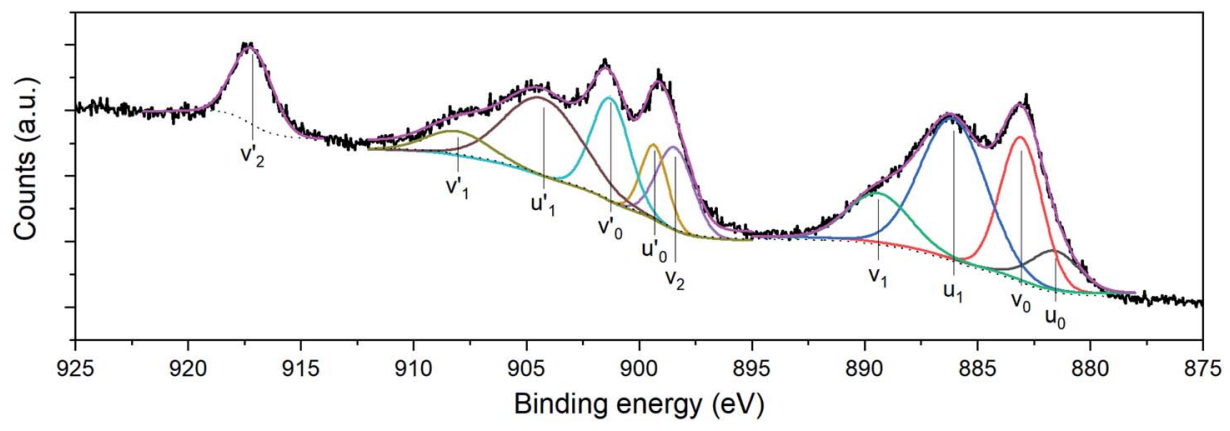

Fig. $5 \mathrm{Ce} 3 d_{3 / 2}$ and $\mathrm{Ce} 3 d_{5 / 2}$ high resolution XPS of GO-CNT-Ce-515. 

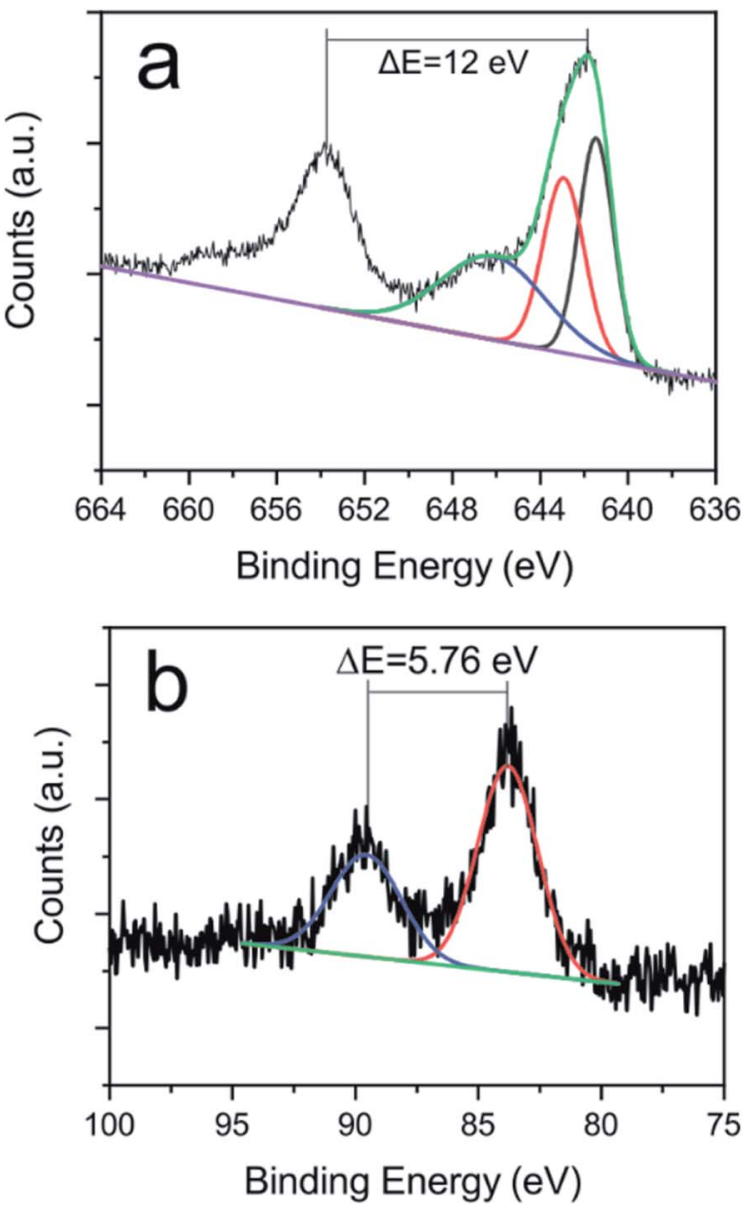

Fig. 6 High resolution (a) Mn $2 p$ and (b) Mn 3s XPS spectra and its fitting curves of sample GO-CNT-Ce-Mn-5152.

XPS spectra of the Mn 2p line (Fig. 6a) is complex because of the presence of a multiplet structure. ${ }^{74}$ The $\mathrm{Mn} 2 \mathrm{p}_{3 / 2}$ spectra is characteristic of the $\mathrm{Mn}_{3} \mathrm{O}_{4}$ and $\mathrm{MnO}$ compounds, having peaks at ca. $641.5 \mathrm{eV}, 642.9 \mathrm{eV}$ and $646.1 \mathrm{eV}{ }^{31,74}$ The doublet separation of the $\mathrm{Mn} 2 \mathrm{p}_{1 / 2}$ and $\mathrm{Mn} 2 \mathrm{p}_{3 / 2}$ spin orbit $(12 \mathrm{eV})$ is also in agreement with these phases. The similar binding energies of Mn $2 \mathrm{p}_{3 / 2}$ peaks hinders the obtainment of the compositional percentages of the compounds. Fortunately, there is a characteristic satellite of $\mathrm{Mn}^{2+}$ and splitting of the Mn 3s line (Fig. 6b) that depends on the oxidation state. ${ }^{75}$ This splitting in the measured spectra corroborates the presence of $\mathrm{MnO}$ and $\mathrm{Mn}_{3} \mathrm{O}_{4}$ phases $^{75}$ which is consistent with HRTEM analyses revealing interplanar distances compatible with both oxide phases. From the Mn 3s XPS spectrum it can be determined that the sample contains a mixture of $\mathrm{Mn}^{2+}(62 \%)$ and $\mathrm{Mn}^{3+}(38 \%)$ states, therefore, having a mixture of $\mathrm{MnO}\left(43 \% \mathrm{Mn}^{2+}\right)$ with $\mathrm{Mn}_{3} \mathrm{O}_{4}$ $\left(19 \% \mathrm{Mn}^{2+}\right.$ and $\left.38 \% \mathrm{Mn}^{3+}\right)$. It is worth noticing that neither peaks related to $\mathrm{Ce}-\mathrm{Mn}$ bonds nor mixed phases have been recorded, pointing out to the main formation of separated cerium and manganese oxides, and a negligible incorporation of $\mathrm{Mn}$ ions into the $\mathrm{CeO}_{2}$ structure even during its molten state. This fact is in concordance with TEM-EDX results (Fig. 2d).

To continue with a deeper study of compositional analysis, synchrotron-based FTIRM studies were performed. Despite the low quantity of material contained in the films, intense synchrotron light source allowed to identify various characteristic bands in the trans-reflection operation mode. Fig. 7 shows the SR-FTIRM of representative samples. GO-raw spectrum has peaks at $810 \mathrm{~cm}^{-1}$ (attributed to $\mathrm{C}-\mathrm{H}$ and/or $\mathrm{C}-\mathrm{O}-\mathrm{C}$ groups), $890 \mathrm{~cm}^{-1}$ (C-O-C), $1050 \mathrm{~cm}^{-1}$ (C-O alkoxy), $1210 \mathrm{~cm}^{-1}$ (C-O-C), $1235 \mathrm{~cm}^{-1}(\mathrm{C}-\mathrm{OH})$ and at $1715 \mathrm{~cm}^{-1}(\mathrm{C}=\mathrm{O})$, all of them oxygencontaining groups. The spectrum also reveals a flatten band at $1600 \mathrm{~cm}^{-1}\left(\mathrm{C}=\mathrm{C} \mathrm{sp}^{2}\right) .{ }^{76,77}$ All of the oxygen-containing bands are reduced or disappear in the characteristic spectra of the deposited samples containing GO, proving its reduction during deposition process. CNT-raw spectrum mainly reveals $\mathrm{C}-\mathrm{OH}$ band from the carboxylic functionalization, though $\mathrm{C}=\mathrm{O}$ band is not witnessed, and the $\mathrm{C}=\mathrm{C} \mathrm{sp}^{2}$ bond from the carbon structure appears slightly shifted to lower wavenumbers. ${ }^{78}$ The bands originated from carboxylic groups disappear in the spectra of GO-CNT-Ce-Mn-5152, GO-CNT-Ce-515 and CNT-Ce.

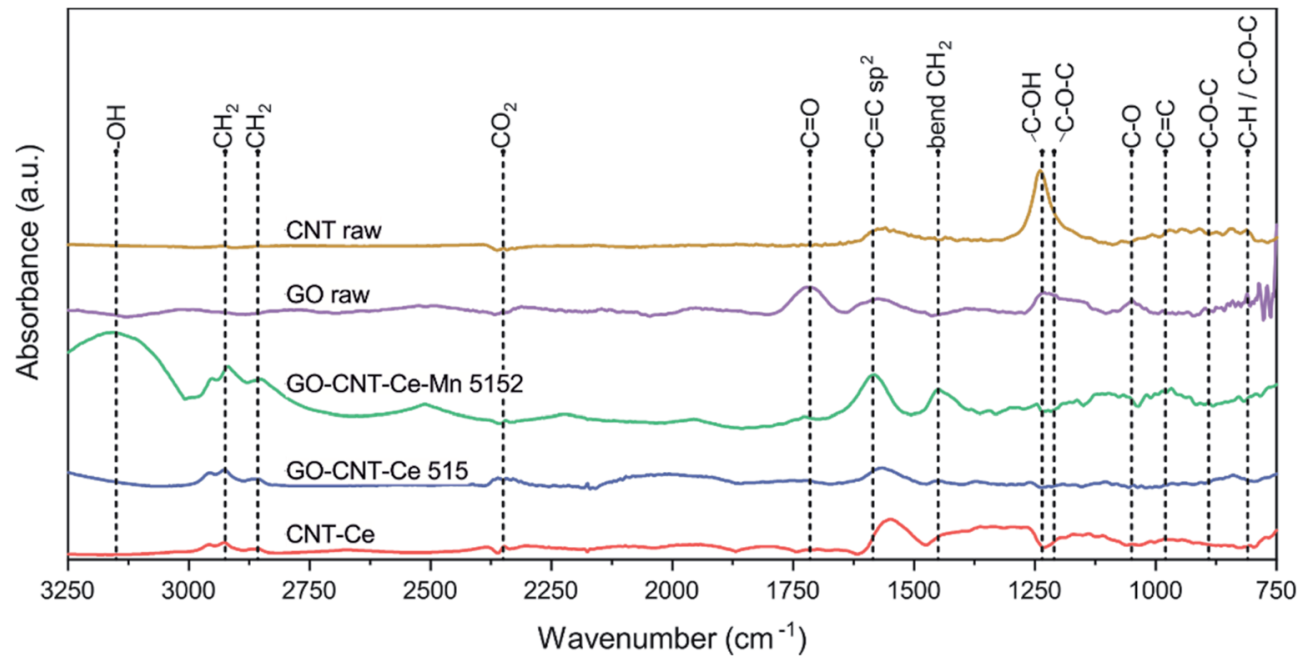

Fig. 7 SR-FTIRM spectra of samples CNT-raw, GO-raw, CNT-Ce, GO-CNT-Ce 515 and GO-CNT-Ce-Mn-5152. 
The spectra of the deposited samples reveal a band at $980 \mathrm{~cm}^{-1}(\mathrm{C}=\mathrm{C})$. The $\mathrm{C}=\mathrm{C} \mathrm{\textrm {sp } ^ { 2 }}$ groups are notable in the spectra of all the deposited samples, showing a broadband in the range $1560-1600 \mathrm{~cm}^{-1}$. The doublet peaks at $2857 \mathrm{~cm}^{-1}$ and $2925 \mathrm{~cm}^{-1}$ are assigned to symmetric and antisymmetric stretching vibrations of $-\mathrm{CH}_{2}$ and the peak at $1450 \mathrm{~cm}^{-1}$ correspond to $-\mathrm{CH}_{2}$ bending. ${ }^{79,80}$ These groups are constituents of alkyl chains, probably formed during reconfiguration of GO to rGO and have been reported to contribute to the electrochemical performance. ${ }^{81}$ The small bands located at $2350 \mathrm{~cm}^{-1}$ are assigned to $\mathrm{CO}_{2}$. The bands above $3150 \mathrm{~cm}^{-1}$ are assigned to $-\mathrm{OH}$ groups.

\subsection{Electrochemical characterization}

Electrochemical analyses were performed to study the energy storage of the electrodes fabricated through cyclic voltammetry (CV) in the $0-0.8 \mathrm{~V}$ voltage window and $10-150 \mathrm{mV} \mathrm{s}^{-1}$ sweep rate ranges. Fig. 8a shows typical voltammograms for the different sweep rates. The peak present at $c a .0 .8 \mathrm{~V}$ is due to the oxygen-evolution reaction in the aqueous electrolyte. The recorded voltammograms show quasi-rectangular shape and no presence of prominent peaks, attributed to redox reactions. This behaviour points to capacitive (surface) processes as main charge storage mechanisms in the electrodes. ${ }^{\mathbf{8 2 , 8 3}}$ To obtain more proves of the surface dominance mechanism, the capacitance contribution of surface and diffusion-controlled charge storage processes are differentiated. In surface-dominant reactions, response current varies linearly with the sweep rate $(s)$. By contrast, processes controlled by semi-infinite diffusion (bulk) are proportional to the square root of the sweep rate. ${ }^{9}$ Therefore, the current can be expressed as

$$
i(s, V)=i_{\text {cap }}+i_{\text {diff }}=k_{1} s+k_{2} s^{1 / 2}
$$

Taking this into account, $k_{1}$ and $k_{2}$ constants can be determined from the fitting of $i(s, V) / s^{1 / 2}$ versus $s^{1 / 2}$, hence differentiating the capacitive current contribution from that of diffusion-controlled processes. Fig. $8 \mathrm{~b}$ and c present the voltammograms of the electrodes GO-Ce and GO-CNT-Ce-Mn5152 , respectively, for $100 \mathrm{mV} \mathrm{s}^{-1}$ sweep rate with highlighted region of the capacitive contribution from the total current measured. Accordingly, Fig. 8d shows the percentage of the capacitive contribution for all the samples. Regarding GO-CNTCe-Mn-5155, $k_{1}$ and $k_{2}$ terms might not be correctly calculated due to lack of linearity of eqn (2) in most of voltage values. Thus, no simple mechanism for the origin of the sweep-rate dependence can be determined. A reported explanation of this behaviour is called the "porous-electrode" effect. The signal may become attenuated in pores having finite resistance leading to a progressively increase of iR-drop in them. Therefore, local response current will become attenuated and cannot be expressed as a simple relation between current and $s$ or $s^{1 / 2} \cdot{ }^{84,85}$ Thus, the surface percentage could be underestimated. Despite the behaviour of this specific sample, in the majority of cases, the main contributions are surface processes. Interestingly, the addition of Mn oxides and CNT increases the diffusive component despite their pseudocapacitive behaviour ${ }^{4}$ and porosity increase, ${ }^{\mathbf{4 6}}$ respectively. Considering the major
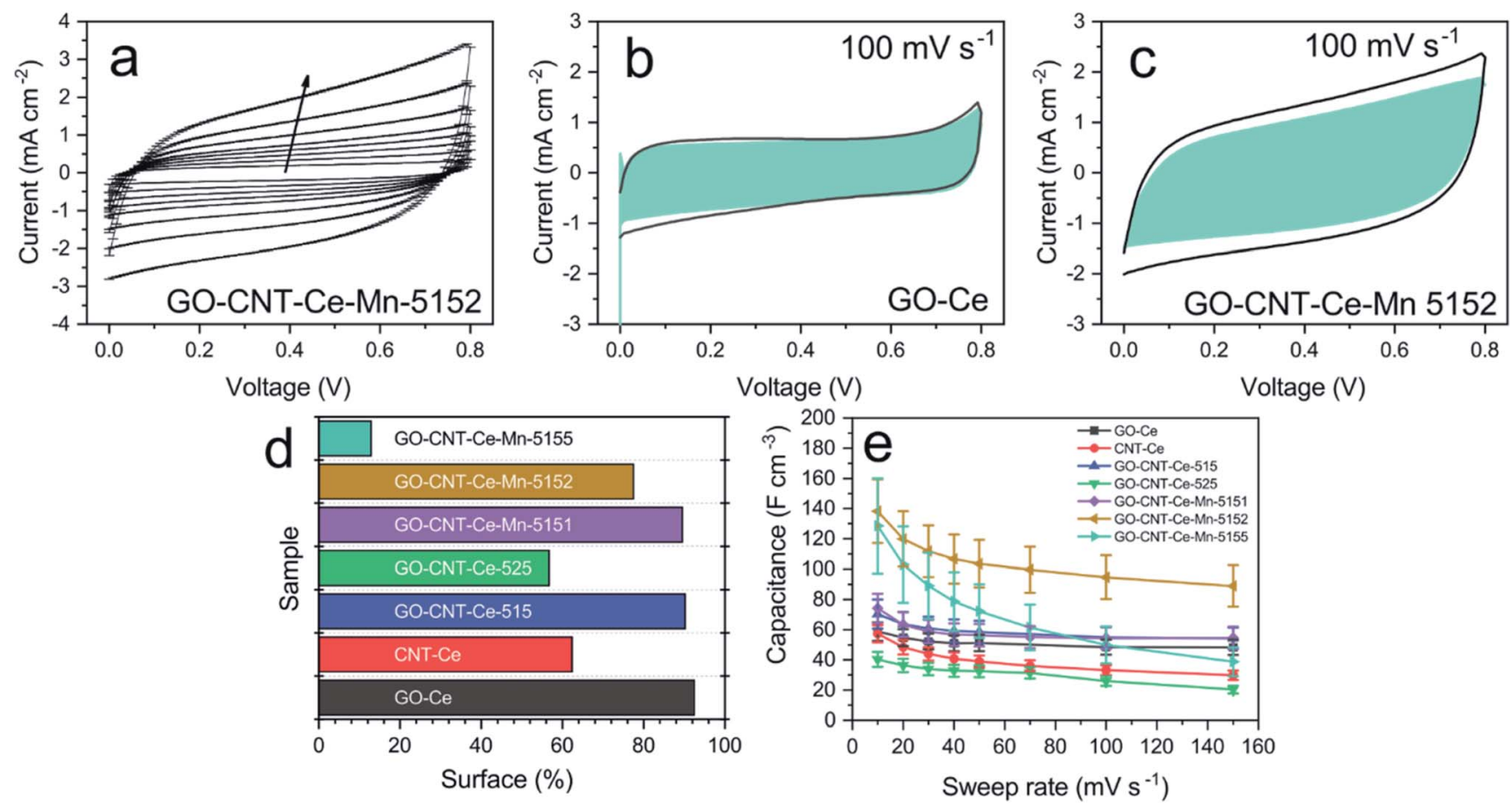

Fig. 8 (a) CV curves of sample GO-CNT-Ce-Mn-5152 (arrow indicates the increasing sweep rate); CV of samples (b) GO-Ce and (c) GO-CNTCe-Mn-5152 at $100 \mathrm{mV} \mathrm{s}^{-1}$ with surface capacitance contribution highlighted in blue; (d) percentage of surface contribution in capacitance of all fabricated samples at $100 \mathrm{mV} \mathrm{s}^{-1}$; (e) volumetric capacitance vs. sweep rate of all the fabricated samples. 
contribution of surface process and the quasi-rectangular shape of the voltammograms, the volumetric capacitance $\left(C_{\mathrm{v}}\right)$ of the electrodes can be calculated from $\mathrm{CV}$ data at different sweep rates following the equation

$$
C_{\mathrm{v}}=\frac{\oint I(V) \mathrm{d} V}{2 \Delta V s v}
$$

where the integral represents the area enclosed of the CV plot (anodic-cathodic charges), $\Delta V$ is the voltage window range $(0.8 \mathrm{~V}), s$ is the sweep rate and $v$ represents the volume of the active material (area $\times$ thickness). Fig. 8e shows the volumetric capacitance at different sweep rates for all the different electrodes. Comparing the capacitance measured at $10 \mathrm{mV} \mathrm{s}^{-1}$, both GO-Ce and CNT-Ce disclose $c a .60 \mathrm{~F} \mathrm{~cm}^{-3}$, although the sample composed of CNT reveals lower storage of energy for higher sweep rates, in agreement with the lower capacitive surface contribution from the total current presented in Fig. 8d. This result supports a previous work revealing that the addition of CNTs to GO-NiO system increases the diffusive process, reducing the capacitance in the fast processes produced at high sweep rates. ${ }^{46}$ The addition of 1 wt\% of CNTs to GO-Ce dispersion increases slightly the capacitance of the obtained electrode to $c a .70 \mathrm{~F} \mathrm{~cm}^{-3}$. The CNTs establish spacing between GO sheets preventing re-stacking and increasing porosity, besides producing an enhancement of the electron transport. ${ }^{48}$ The addition of a higher concentration of CNTs ( $2 \mathrm{wt} \%$ ) reduces the capacitance to $40 \mathrm{~F} \mathrm{~cm}^{-3}$, because of the increased agglomeration of the materials, ${ }^{48}$ reducing the pathway for the electrolyte. Using the GO-CNT-Ce composite with the best capacitance ( $1 \mathrm{wt} \%$ of CNT), the addition of manganese oxides increases its electrochemical properties in all the cases. With the addition of manganese acetate with only $1 / 5$ concentration of that of $\mathrm{CeO}_{2}$ NPs, the capacitance reaches $c a .75 \mathrm{~F} \mathrm{~cm}^{-3}$, which is slightly higher than GO-CNT-Ce composite. Increasing the amount of manganese oxide precursor with $2 / 5$ and $5 / 5 \mathrm{Ce} / \mathrm{Mn}$ concentration, the capacitance boosts to $c a .140 \mathrm{~F} \mathrm{~cm}^{-3}$ and $130 \mathrm{~F} \mathrm{~cm}^{-3}$, respectively. Taking into consideration the experimental error (caused essentially by the film thickness), both values can be considered comparable. It is significant the higher decay in the capacitance at higher sweep rates for the electrode obtained with the highest amount of manganese precursor. As already observed, this behaviour is due to an increase of the diffusive contribution suggesting either the movement limitation of electrolyte ions or to the extrinsic pseudocapacitive nature of manganese oxides ( $\mathrm{MnO}$ and $\left.\mathrm{Mn}_{3} \mathrm{O}_{4}\right){ }^{21-24,31}$ It is worth noting that the volumetric capacitance achieved in this work, by co-deposition of $\mathrm{CeO}_{x}$ and $\mathrm{MnO}_{x}$ oxides, is considerably higher than the best one already obtained by RIMAPLE fabrication of GO-based micrometric thickness electrodes in combination with either $\mathrm{NiO}$ or $\mathrm{ZnO}$ NPs (ca. $\left.30 \mathrm{~F} \mathrm{~cm}^{-3}\right){ }^{46,86}$ Furthermore, the study of the volumetric capacitance dependence on electrode's thickness reveals almost no changes (Fig. S8a $\dagger$ ). This result proves that the porosity is preserved even at thicker electrodes, having the electrolyte ions good diffusion towards the active surface. Even though, there is a slight increase of capacitance at lower scan rates for thicker electrodes which is attributable to the larger active surface involved. On the contrary, there is a decrease at higher scan rates caused by slight hindering of diffusion processes (Fig. S8b $\dagger$ ).

Galvanostatic charge-discharge (GCD) measurements were performed to the electrodes in a current density range of $0.2-2 \mathrm{~mA} \mathrm{~cm}^{-2}$. A typical charge-discharge curve is presented in Fig. 9a, showing the quasi-linear voltage response with time that generates the characteristic triangular shape of capacitive materials. It can be also observed that with the increasing current, the charge/discharge time decreases. The coulombic efficiency (discharge/charge time relation) is around $98 \%$, hence no charge is practically lost during operation. The volumetric capacitance can be calculated from the GCD data using

$$
C_{\mathrm{v}}=\frac{I \Delta t_{\mathrm{disch}}}{\Delta V d_{\mathrm{thk}}}
$$

where $I$ is the applied current density, $\Delta t_{\text {disch }}$ is the discharge time, $\Delta V$ is the voltage window range $(0.8 \mathrm{~V})$ and $d_{\text {thk }}$ is the thickness of the deposited film. As shown in Fig. 9b, the values of capacitance obtained are compatible with the ones from CV data, reaching similar values for $0.2 \mathrm{~mA} \mathrm{~cm}^{-2}$ applied current to the ones at $10 \mathrm{mV} \mathrm{s}^{-1}$ sweep rate. There is also a decay in capacitance as applied current density increases, accounting for faster processes with less charge stored.
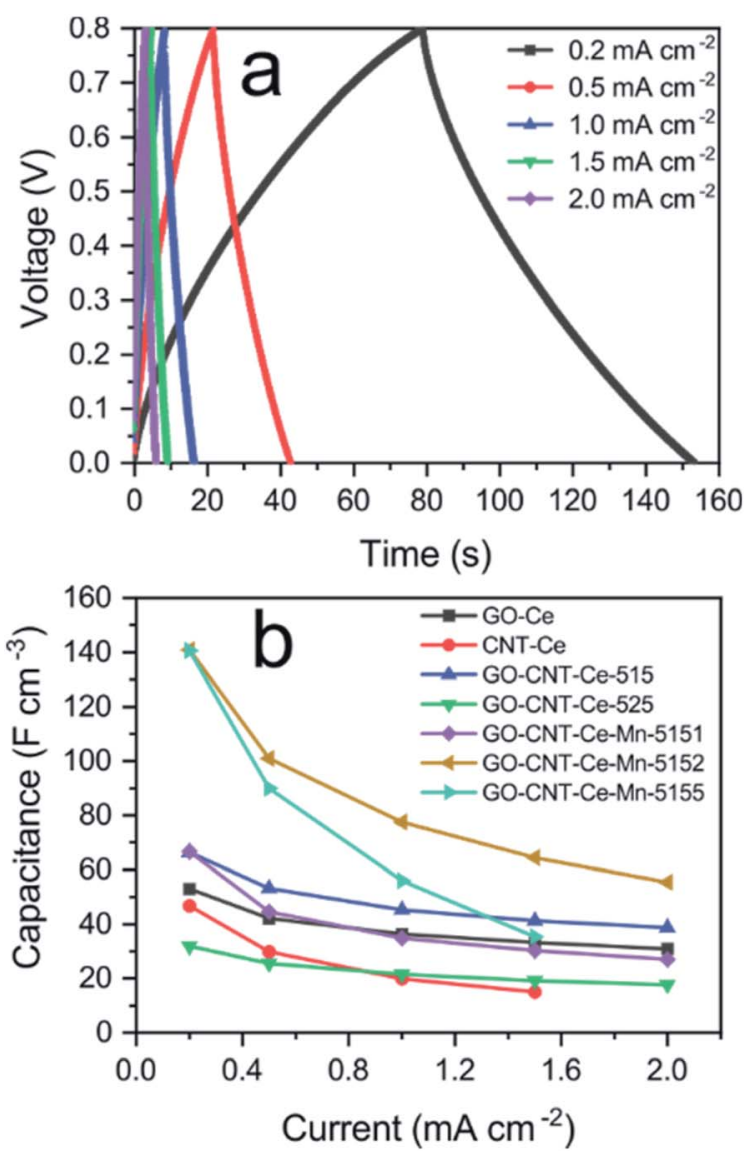

Fig. 9 (a) Volumetric capacitance vs. applied current density of all the fabricated samples; (b) galvanostatic charge-discharge data of GOCNT-Ce-Mn-5152 for different applied currents. 

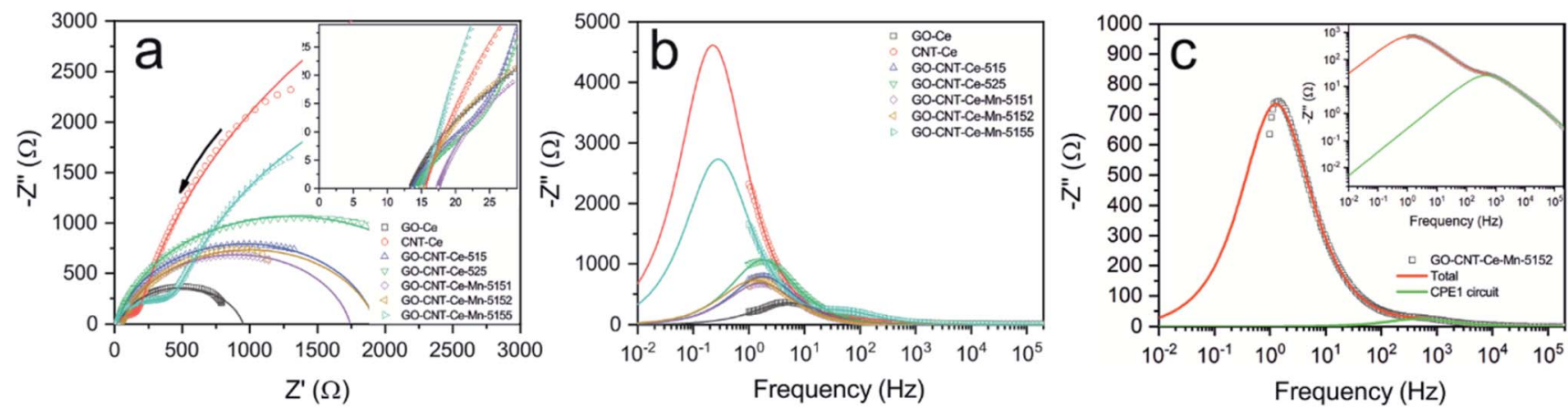

Fig. 10 (a) Nyquist plot of EIS measurement (points) and simulated from equivalent circuit (lines) of all the fabricated samples (arrow indicates the direction of increasing frequency). Inset details the high frequency range. Plot of imaginary part of impedance as function of the frequency (b) of the fabricated samples; and (c) of sample GO-CNT-Ce-Mn-5152 with deconvoluted CPE1 contribution. Inset is presented with logarithmic scale on the imaginary impedance axis.

The best values of volumetric capacitance acquired with either CV or GCD, are comparable to the ones reported in the literature ${ }^{6,87}$ and almost one order of magnitude higher than GO-CNT-NiO electrodes also obtained with RIMAPLE. ${ }^{46}$

Additional studies of the electrodes were performed with electrochemical impedance spectroscopy (EIS) measurements. Fig. 10a presents the Nyquist plot for all the samples, where the expected behaviour of carbon-based porous electrodes can be observed. At high and middle frequencies, EDL and charge transfer reactions take place, producing a partial semicircle loop. At low frequency, diffusion processes are predominant, generating the quasi-linear straight line in some cases and partial semicircle of finite diffusion in the rest. ${ }^{88}$ All the curves were fitted using the equivalent circuit represented in Fig. S9† (modified Randles cell), and its plot is presented as continuous lines in Fig. 10a. The equivalent circuit is composed of three resistances: $R 1$ as equivalent series resistance (ESR), $R 2$ as charge-transfer resistance and $R 3$ accounting for leakages. The two other components are constant phase elements (CPE). The impedance of these elements is described as

$$
Z_{\mathrm{CPE}}=\frac{1}{Y(j \omega)^{n}}
$$

In this case, $Y$ represents the capacitance and $n$ index has a value between 0 and 1 . A CPE with $n$ value of 1 indicates an ideal capacitor, whereas lower values account for material inhomogeneity. CPE1 represents the double layer charge storage processes and CPE2 adds the pseudocapacitive contribution generated by redox mechanisms. ${ }^{45}$ Table S2 $\uparrow$ summarizes the values of the elements for the equivalent circuit fitting of the EIS data. The ESR or R1, that can also be determined in the cut of the horizontal axis in the inset of Fig. 10a, is similar in all the electrodes (about $15 \Omega$ ). This resistance is the sum of the intrinsic resistance of electrodes and that of the electrolyte. The $R 2$ (charge-transfer resistance) values are similar in all the electrodes except in CNT-Ce and GO-CNT-Ce-Mn-5155, which show about one order of magnitude higher values. Those electrodes presented higher decay of capacitance at higher sweep rates (Fig. 8e). This higher resistance accounts for a slower storage of energy, decreasing the performance in fast sweep rates. Warburg impedance elements were negligible in the fitted model, pointing to $R 2$ element as the one including the contribution of electrolyte diffusion in the studied frequency range. $R 3$ (leakage resistance) values are in the order of $\mathrm{k} \Omega$ revealing low charge leakage. Regarding CPE, all $n$ values are higher than 0.82 confirming almost ideal capacitors. It is important to notice the significant increase of capacitance values for CPE2 (pseudocapacitance) in the samples including Mn oxides, proving the high pseudocapacitance incorporated to the electrodes through these compounds.

For a deeper study of the impedance spectra, a key parameter is the relaxation time constant, $\tau$, that acts as a factor of merit and it is characteristic of each sample. The frequently used method to determine $\tau$ is with the phase Bode plot, with the corresponding time on the cut at $45^{\circ}$. However, this method of

Table 2 Relaxation time constant from CPE1 and CPE2 for the different samples

\begin{tabular}{lll}
\hline & $\begin{array}{l}\text { Relaxation time constant } \\
\text { from CPE1 (ms) }\end{array}$ & $\begin{array}{l}\text { Relaxation time } \\
\text { constant from CPE2 (ms) }\end{array}$ \\
\hline GO-Ce & 2 & 200 \\
CNT-Ce & 7 & 4500 \\
GO-CNT-Ce-515 & 2 & 550 \\
GO-CNT-Ce-525 & 1 & 550 \\
GO-CNT-Ce-Mn-5151 & 2 & 600 \\
GO-CNT-Ce-Mn-5152 & 2 & 800 \\
GO-CNT-Ce-Mn-5155 & 18 & 3600
\end{tabular}


determination is not applicable when the series resistance to the parallel $R \mid \mathrm{CPE}$ circuit has a similar order of magnitude in resistance values. ${ }^{89}$ In these cases, the difficulties can be overcome with the representation of the impedance imaginary part as function of the frequency. The resistance components have purely real impedance component, and they do not intervene in this plot. Therefore, the contribution comes merely from the capacitive components, where the relaxation time constant can be obtained through the inverse of frequency at local maximum. Fig. 10b presents the plot of the impedance imaginary component of all the measured data (points) and simulation with the equivalent circuit transfer function (lines). The low frequency maximum, which corresponds to pseudocapacitive relaxation time (CPE2), is clearly determined. It is important to take into account that two samples (CNT-Ce and GO-CNT-Ce-Mn-5155) have this maximum below $1 \mathrm{~Hz}$, which is the minimum experimentally measured frequency and its values are calculated. Because of the high signal of the low frequency maximum, the relaxation time constant related to faster processes found at higher frequency values, cannot be directly determined. However, a capacitor behaves as a short circuit when excited at frequencies much higher than their characteristic one. Therefore, the impedance contribution of the rest of the elements can be calculated using the obtained equivalent circuit parameters and considering a circuit without neither CPE2 nor $R 3$, obtaining the relaxation time constant related to CPE1. Fig. 10c presents the deconvolution of one specific case (GO-CNT-Ce-
Mn-5152) to show the different contributions. A bulge can be differentiated, specially using logarithmic scale on the imaginary impedance axis (inset of Fig. 10c). Table 2 shows the obtained relaxation time constants related to the two different processes involved (EDL and pseudocapacitance). Relaxation time constants related to CPE1 are similar in all the cases, in the order of the millisecond, except for CNT-Ce and GO-CNT-CeMn-5155, which have slightly higher times. On the other hand, relaxation times related to CPE2 exhibit significant variations. The addition of CNT to GO-Ce increases the characteristic time, and the addition of Mn oxides induces an even higher increase of characteristic times, accounting for the remarkable increase of the pseudocapacitance and diffusion processes. The characteristic time of CNT-Ce is also higher than the GO-Ce due to the increase of porosity between CNTs, which produces aggregated oxide structures that lead to slower charge transport mechanisms.

\subsection{Device fabrication and performance study}

The working performance of the best volumetric capacitance electrode was investigated by assembling a symmetric electrochemical capacitor with two GO-CNT-Ce-Mn-5152 electrodes. Fig. 11a shows the typical voltammograms for various sweep rates, presenting similar shape to the single electrode (Fig. 8a). The volumetric capacitance at different sweep rates (CV) and different applied current (GCD) can be found in Fig. 11b and c, respectively, reaching $c a .25 \mathrm{~F} \mathrm{~cm}^{-3}$ in both cases. As expected,
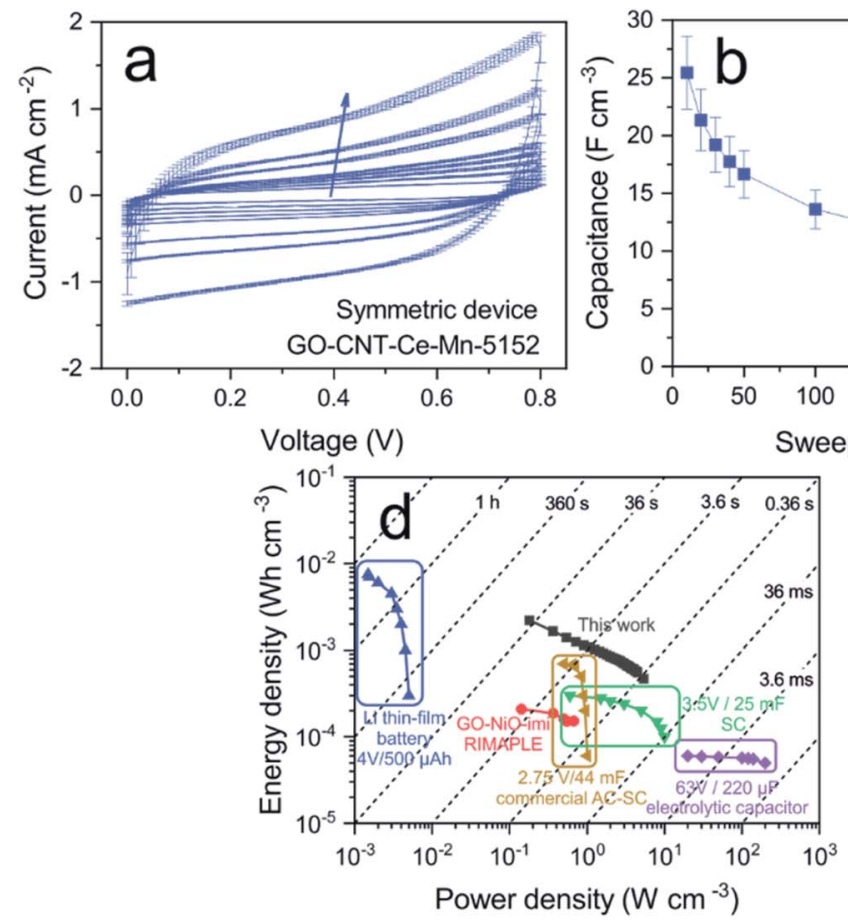
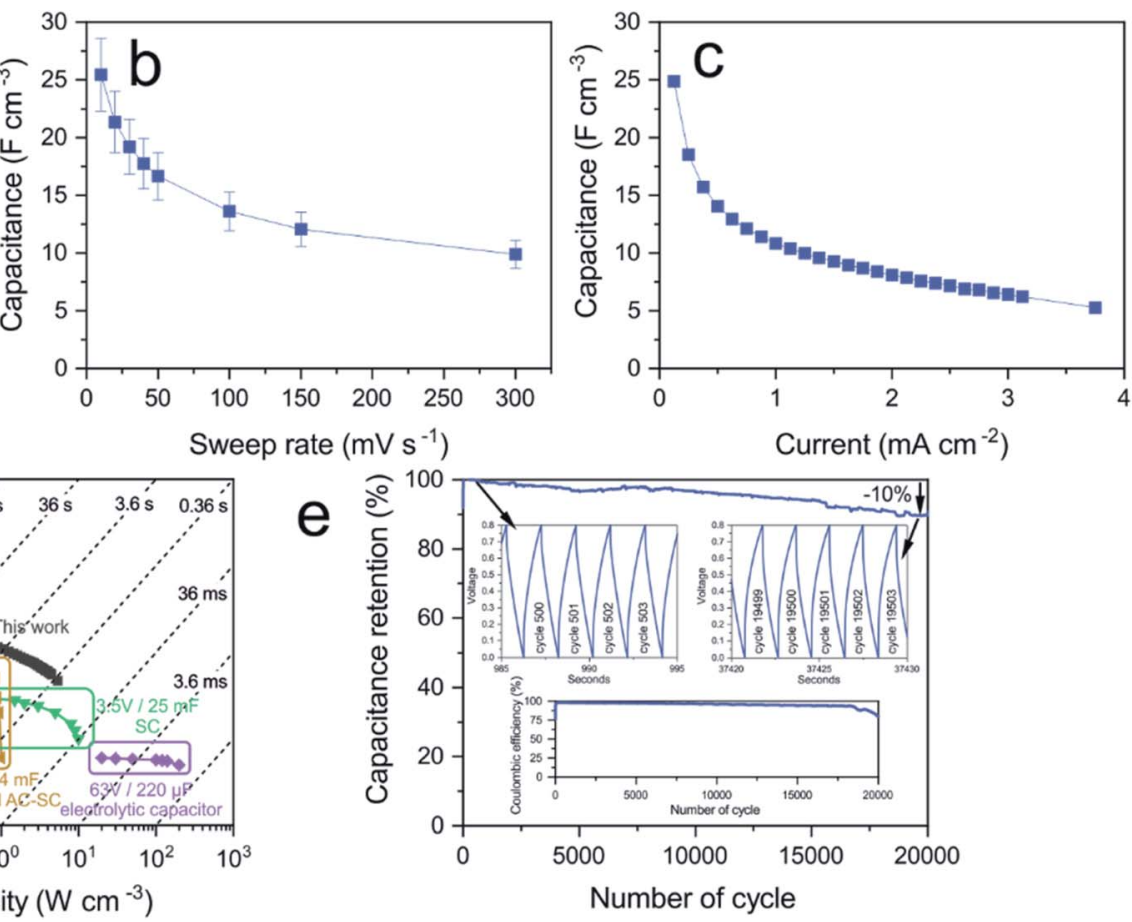

Fig. 11 (a) CV curves of symmetric device GO-CNT-Ce-Mn-5152 (arrow indicates the increasing sweep rate), (b) volumetric capacitance vs. sweep rate, (c) volumetric capacitance vs. applied current, (d) Energy and power densities of the fabricated SC compared with commercially available energy storage systems. Data for the lithium battery, $3.5 \mathrm{~V} / 25 \mathrm{mF}$ supercapacitor (SC) and $6.3 \mathrm{~V} / 220 \mu \mathrm{F}$ electrolytic capacitor, ${ }^{90}$ data for the $2.75 \mathrm{~V} / 44 \mathrm{mV}$ activated carbon supercapacitor (AC-SC) ${ }^{91}$ and $\mathrm{GO}-\mathrm{NiO}$-imi symmetric device. ${ }^{45}$ (e) Capacitance retention with chargedischarge zoomed regions (superior insets) and coulombic efficiency (inferior inset). 
the values are lower than with a single electrode due to the fact that both capacitors are in series. The Ragone plot (volumetric energy vs. power densities), shown in Fig. 11d, allows the evaluation of the global performance of the device. For the calculation, the total volume of the active material has been used. A comparison of different energy storage devices is also presented. ${ }^{45,90,91}$ It is important to emphasize the increase in both energy and power densities as compared to previous RIMAPLE symmetric N-doped rGO-NiO device, obtained with the same fabrication technique. As witnessed, the fabricated $\mathrm{CeO}_{x}-\mathrm{MnO}_{x^{-}}$ rGO-CNT capacitor exhibits higher power and energy densities, up to $5.4 \mathrm{~W} \mathrm{~cm}^{-3}$ and $2.2 \mathrm{~mW} \mathrm{~h} \mathrm{~cm}^{-3}$, respectively. Despite that submicrometric thickness electrodes obtained by RIMAPLE method are reported having comparable or higher storage capabilities, ${ }^{45,61,92}$ it has to be taken into account that the performance of such thin electrodes will not be scalable without losses due to microstructural rearrangements that hinders the electrolyte diffusion and interaction with active material. ${ }^{93}$ The stability of the device was also studied by galvanostatic chargedischarge cycling at ca. $2 \mathrm{~mA} \mathrm{~cm} \mathrm{~cm}^{-2}$ current density (Fig. 11e). The capacitance retention and the coulombic efficiency decay ca. $10 \%$ after 20000 cycles, maintaining the triangular chargedischarge shape practically unaltered (Fig. 11e, inset). The decay is presumably related to electrolyte drying process as previously suggested for poorly sealed devices. Further work will be focused on both the enhancement of the EC sealing and post-mortem analyses of the electrodes. Even though, the stability has been proved, doubling the number of cycles from previous experiments. ${ }^{45}$

\section{Conclusions}

The presented hybrid electrodes developed by RIMAPLE laser technique show excellent performance for electrochemical supercapacitors. Through UV laser radiation, a myriad of photochemical and photothermal processes occur inducing the reduction of GO and the crystallization of metal oxides on the surface of carbon nanomaterials. The decomposition of manganese acetate through laser processing is demonstrated with the formation of different oxides, $\mathrm{MnO}$ and $\mathrm{Mn}_{3} \mathrm{O}_{4}$, besides chemical modification of ceria nanoparticles, submitted to melting-coalescence-dewetting mechanisms, changing their crystallographic phase to $\mathrm{Ce}_{2} \mathrm{O}_{3}$. The thorough morphological, compositional and electrochemical analyses of the electrodes provide proper understanding of the composite fabricated and the mechanisms involved in charge storage. The electrodes fabricated with rGO, MWCNT, Ce and Mn oxides combine EDL and pseudocapacitance storage mechanisms. The maximum volumetric capacitance of $140 \mathrm{~F} \mathrm{~cm}^{-3}$ obtained is significantly higher than in previous works using RIMAPLE technique with NiO NPs. Finally, the assembly of a symmetric electrochemical capacitor proves the practical use of the developed hybrid electrodes standing out their remarkable high stability upon cycling. These outstanding results open the door to innovative and versatile development of supercapacitors using laser deposition techniques, with combinations of different metal oxides either in nanoparticles or in ionic forms (dissolved metal organic precursors), allowing the formation of binary metal oxides systems with new properties besides the use of different combinations of carbon nanomaterials.

\section{Conflicts of interest}

There are no conflicts to declare.

\section{Acknowledgements}

The authors thank the financial support of the Spanish Ministry of Economy, Industry and Competitiveness under the project ENE2017-89210-C2-1-R and support from AGAUR of Generalitat de Catalunya through projects 2017 SGR 1086 and 2017 SGR 1771. PGL thanks the financial support of the Spanish Ministry of Economy, Industry and Competitiveness through the grant BES-2017-081652 for the formation of scientific researchers. ICMAB acknowledges financial support from the Spanish Ministry of Economy and Competitiveness, through Severo Ochoa FUNFUTURE (CEX2019-000917-S). The FTIRM experiments were performed at MIRAS beamline at ALBA Synchrotron with the collaboration of ALBA staff. We acknowledge support of the publication fee by the CSIC Open Access Publication Support Initiative through its Unit of Information Resources for Research (URICI).

\section{References}

1 E. Pomerantseva, F. Bonaccorso, X. Feng, Y. Cui and Y. Gogotsi, Science, 2019, 366, 1-12.

2 Poonam, K. Sharma, A. Arora and S. K. Tripathi, Journal of Energy Storage, 2019, 21, 801-825.

3 J. Yan, Q. Wang, T. Wei and Z. Fan, Adv. Energy Mater., 2014, 4, 1-43.

4 A. González, E. Goikolea, J. A. Barrena and R. Mysyk, Renewable Sustainable Energy Rev., 2016, 58, 1189-1206.

5 L. Li, J. Zhang, Z. Peng, Y. Li, C. Gao, Y. Ji, R. Ye, N. D. Kim, Q. Zhong, Y. Yang, H. Fei, G. Ruan and J. M. Tour, Adv. Mater., 2016, 28, 838-845.

6 M. Wuttke, Z. Liu, H. Lu, A. Narita and K. Müllen, Batteries Supercaps, 2019, 2, 929-933.

7 X. Huang, X. Qi, F. Boey and H. Zhang, Chem. Soc. Rev., 2012, 41, 666-686.

8 A. Borenstein, O. Hanna, R. Attias, S. Luski, T. Brousse and D. Aurbach, J. Mater. Chem. A, 2017, 5, 12653-12672.

9 V. Augustyn, P. Simon and B. Dunn, Energy Environ. Sci., 2014, 7, 1597-1614.

10 M. Zhi, C. Xiang, J. Li, M. Li and N. Wu, Nanoscale, 2013, 5, 72-88.

11 N. Padmanathan and S. Selladurai, RSC Adv., 2014, 4, 65276534.

12 N. Maheswari and G. Muralidharan, Energy and Fuels, 2015, 29, 8246-8253.

13 K. Prasanna, P. Santhoshkumar, Y. N. Jo, I. N. Sivagami, S. H. Kang, Y. C. Joe and C. W. Lee, Appl. Surf. Sci., 2018, 449, 454-460. 
14 Y. Luo, T. Yang, Q. Zhao and M. Zhang, J. Alloys Compd., 2017, 729, 64-70.

15 D. Deng, N. Chen, X. Xiao, S. Du and Y. Wang, Ionics, 2017, 23, 121-129.

16 R. Murugan, G. Ravi, R. Yuvakkumar, S. Rajendran, N. Maheswari, G. Muralidharan and Y. Hayakawa, Ceram. Int., 2017, 43, 10494-10501.

17 S. J. Zhu, J. Q. Jia, T. Wang, D. Zhao, J. Yang, F. Dong, Z. G. Shang and Y. X. Zhang, Chem. Commun., 2015, 51, 14840-14843.

18 A. K. Singh, D. Sarkar, K. Karmakar, K. Mandal and G. G. Khan, ACS Appl. Mater. Interfaces, 2016, 8, 2078620792.

19 Y. Wang, H. Wei, H. Lv, Z. Chen, J. Zhang, X. Yan, L. Lee, Z. M. Wang and Y. L. Chueh, ACS Nano, 2019, 13, 1123511248.

20 H. Jia, Y. Cai, X. Zheng, J. Lin, H. Liang, J. Qi, J. Cao, J. Feng and W. Fei, ACS Appl. Mater. Interfaces, 2018, 10, 3896338969.

21 S. Nagamuthu, S. Vijayakumar and G. Muralidharan, Energy and Fuels, 2013, 27, 3508-3515.

22 G. Lee, D. Kim, J. Yun, Y. Ko, J. Cho and J. S. Ha, Nanoscale, 2014, 6, 9655-9664.

23 S. Chen, L. Wang, M. Huang, L. Kang, Z. Lei, H. Xu, F. Shi and Z. H. Liu, Electrochim. Acta, 2017, 242, 10-18.

24 T. Xiong, W. S. V. Lee, X. Huang and J. M. Xue, J. Mater. Chem. A, 2017, 5, 12762-12768.

25 G. He, H. Fan, L. Ma, K. Wang, C. Liu, D. Ding and L. Chen, Appl. Surf. Sci., 2016, 366, 129-138.

26 J. Liu, J. Jiang, C. Cheng, H. Li, J. Zhang, H. Gong and H. J. Fan, Adv. Mater., 2011, 23, 2076-2081.

27 X. Sun, Q. Li, Y. Lü and Y. Mao, Chem. Commun., 2013, 49, 4456-4458.

28 D. Sarkar, G. G. Khan, A. K. Singh and K. Mandal, J. Phys. Chem. C, 2013, 117, 15523-15531.

29 Y. Gu, J. Cai, M. He, L. Kang, Z. Lei and Z. H. Liu, J. Power Sources, 2013, 239, 347-355.

30 F. Li, G. Li, H. Chen, J. Q. Jia, F. Dong, Y. B. Hu, Z. G. Shang and Y. X. Zhang, J. Power Sources, 2015, 296, 86-91.

31 R. Dong, Q. Ye, L. Kuang, X. Lu, Y. Zhang, X. Zhang, G. Tan, Y. Wen and F. Wang, ACS Appl. Mater. Interfaces, 2013, 5, 9508-9516.

32 G. Wang, Z. Ma, G. Zhang, C. Li and G. Shao, Electrochim. Acta, 2015, 182, 1070-1077.

33 H. Zhang, J. Gu, J. Tong, Y. Hu, B. Guan, B. Hu, J. Zhao and C. Wang, Chem. Eng. J., 2016, 286, 139-149.

34 G. P. Ojha, B. Pant, S. J. Park, M. Park and H. Y. Kim, J. Colloid Interface Sci., 2017, 494, 338-344.

35 R. Rajagopal and K. S. Ryu, ChemElectroChem, 2018, 5, 22182227.

36 R. Peng, H. Zhang, L. Gui, Y. Zheng, Z. Wu, Y. Luo and P. Yu, Electrochim. Acta, 2019, 319, 95-100.

37 C. Liu, H. Sun, J. Qian, Z. Chen, F. Chen, S. Liu, Y. Lv, X. Lu and A. Chen, J. Alloys Compd., 2017, 722, 54-59.

38 R. You, Y. Liu, Y. Hao, D. Han, Y. Zhang and Z. You, Adv. Mater., 2019, 1901981, 1-22.
39 X. Y. Fu, Z. Di Chen, Y. L. Zhang, D. D. Han, J. N. Ma, W. Wang, Z. R. Zhang, H. Xia and H. B. Sun, Nanoscale, 2019, 11, 9133-9140.

40 D. Bäuerle, Laser Processing and Chemistry, 2011, vol. 208.

41 S. M. O'Malley, J. Tomko, Á. Pérez del Pino, C. Logofatu and E. György, J. Phys. Chem. C, 2014, 118, 27911-27919.

42 E. György, C. Logofatu, Á. Pérez del Pino, A. Datcu, O. Pascu and R. Ivan, Ceram. Int., 2018, 44, 1826-1835.

43 R. Ivan, C. Popescu, A. P. del Pino, I. Yousef, C. Logofatu and E. György, J. Mater. Sci., 2019, 54, 3927-3941.

44 A. Queraltó, Á. Pérez del Pino, C. Logofatu, A. Datcu, R. Amade, I. Alshaikh, E. Bertrán, I. Urzica and E. György, J. Alloys Compd., 2017, 726, 1003-1013.

45 Á. Pérez del Pino, M. A. Ramadan, P. García Lebière, R. Ivan, C. Logofatu, I. Yousef and E. György, Appl. Surf. Sci., 2019, 484, 245-256.

46 Á. Pérez Del Pino, M. Rodríguez López, M. A. Ramadan, P. García Lebière, C. Logofatu, I. Martínez-Rovira, I. Yousef and E. György, Phys. Chem. Chem. Phys., 2019, 21, 2517525186.

47 M. A. Steiner and J. M. Fitz-Gerald, Appl. Phys. A: Mater. Sci. Process., 2015, 119, 629-638.

48 X. Cui, R. Lv, R. U. R. Sagar, C. Liu and Z. Zhang, Electrochim. Acta, 2015, 169, 342-350.

49 A. Queraltó, Á. Pérez del Pino, M. De La Mata, J. Arbiol, M. Tristany, X. Obradors and T. Puig, Chem. Mater., 2016, 28, 6136-6145.

50 V. A. Smirnov, A. A. Arbuzov, Y. M. Shul'ga, S. A. Baskakov, V. M. Martynenko, V. E. Muradyan and E. I. Kresova, High Energy Chem., 2011, 45, 57-61.

51 Z. Wan, E. W. Streed, M. Lobino, S. Wang, R. T. Sang, I. S. Cole, D. V. Thiel and Q. Li, Adv. Mater. Technol., 2018, 3, 1-19.

52 A. Vogel and V. Venugopalan, Chem. Rev., 2003, 103, 577644.

53 Á. Pérez del Pino, E. György, L. Cabana, B. Ballesteros and G. Tobias, J. Appl. Phys., 2014, 115, 093501.

54 E. K. Goharshadi, S. Samiee and P. Nancarrow, J. Colloid Interface Sci., 2011, 356, 473-480.

55 J. A. Dean and N. A. Lange, Lange's handbook of chemistry, McGraw-Hill, New York, 1999.

56 J. Y. Hwang, M. F. El-Kady, Y. Wang, L. Wang, Y. Shao, K. Marsh, J. M. Ko and R. B. Kaner, Nano Energy, 2015, 18, 57-70.

57 A. Younis, D. Chu and S. Li, in Functionalized Nanomaterials, ed. M. Akhyar Farrukh, IntechOpen, 2016, pp. 53-68.

58 D. A. Edwards and R. N. Hayward, Can. J. Chem., 1968, 46, 3443-3446.

59 M. A. Mohamed and S. A. Halawy, Thermochim. Acta, 1994, 242, 173-186.

60 N. A. M. Barakat, K. Do Woo, S. G. Ansari, J. A. Ko, M. A. Kanjwal and H. Y. Kim, Appl. Phys. A: Mater. Sci. Process., 2009, 95, 769-776.

61 Á. Pérez del Pino, A. Martínez Villarroya, A. Chuquitarqui, C. Logofatu, D. Tonti and E. György, J. Mater. Chem. A, 2018, 6, 16074-16086. 
62 Á. Pérez del Pino, E. György, C. Logofatu and A. Duta, J. Phys. D: Appl. Phys., 2013, 46, 1-8.

63 A. T. Nelson, D. R. Rittman, J. T. White, J. T. Dunwoody, M. Kato and K. J. McClellan, J. Am. Ceram. Soc., 2014, 97, 3652-3659.

64 S. Debnath, M. R. Islam and M. S. R. Khan, Bull. Mater. Sci., 2007, 30, 315-319.

65 Á. Pérez del Pino, E. György, C. Logofatu, J. Puigmartí-Luis and W. Gao, Carbon, 2015, 93, 373-383.

66 C. Barth, C. Laffon, R. Olbrich, A. Ranguis, P. Parent and M. Reichling, Sci. Rep., 2016, 6, 2-7.

67 Á. Pérez del Pino, A. González-Campo, S. Giraldo, J. Peral, E. György, C. Logofatu, A. J. DeMello and J. Puigmartí-Luis, Carbon, 2018, 130, 48-58.

68 Y. Wu, R. Shu, J. Zhang, Z. Wan, J. Shi, Y. Liu, G. Zhao and M. Zheng, J. Alloys Compd., 2020, 819, 152944.

69 D. Banerjee and H. W. Nesbitt, Geochim. Cosmochim. Acta, 1999, 63, 3025-3038.

70 Z. Zeng, X. Long, H. Zhou, E. Guo, X. Wang and Z. Hu, Electrochim. Acta, 2015, 163, 107-115.

71 L. Qiu, F. Liu, L. Zhao, Y. Ma and J. Yao, Appl. Surf. Sci., 2006, 252, 4931-4935.

72 X. Yu and G. Li, J. Alloys Compd., 2004, 364, 193-198.

73 J. J. Plata, A. M. Márquez and J. F. Sanz, J. Phys. Chem. C, 2013, 117, 14502-14509.

74 M. C. Biesinger, B. P. Payne, A. P. Grosvenor, L. W. M. Lau, A. R. Gerson and R. S. C. Smart, Appl. Surf. Sci., 2011, 257, 2717-2730.

75 F. Müller, R. De Masi, D. Reinicke, P. Steiner, S. Hüfner and K. Stöwe, Surf. Sci., 2002, 520, 158-172.

76 R. Trusovas, G. Račiukaitis, G. Niaura, J. Barkauskas, G. Valušis and R. Pauliukaite, Adv. Opt. Mater., 2016, 4, 3765.

77 X. Mao, J. Xu, X. He, W. Yang, Y. Yang, L. Xu, Y. Zhao and Y. Zhou, Appl. Surf. Sci., 2018, 435, 1228-1236.
78 C. S. Chen, X. H. Chen, L. S. Xu, Z. Yang and W. H. Li, Carbon, 2005, 43, 1660-1666.

79 M. Shateri-Khalilabad and M. E. Yazdanshenas, Cellulose, 2013, 20, 963-972.

80 A. Kaniyoor, T. T. Baby and S. Ramaprabhu, J. Mater. Chem., 2010, 20, 8467-8469.

81 G. Zhao, F. G. Zhao, J. Sun, W. Wang, Y. Lu, W. S. Li and Q. Y. Chen, Carbon, 2015, 94, 114-119.

82 Y. Gogotsi and R. M. Penner, ACS Nano, 2018, 12, 2081-2083.

83 J. Liu, J. Wang, C. Xu, H. Jiang, C. Li, L. Zhang, J. Lin and Z. X. Shen, Adv. Sci., 2018, 5, 1-19.

84 T. C. Liu, W. G. Pell, B. E. Conway and S. L. Roberson, J. Electrochem. Soc., 1998, 145, 1882-1888.

85 E. Barsoukov and J. R. Macdonald, Impedance Spectroscopy Theory, Experiment, and Applications, John Wiley \& Sons, Inc., Hoboken, New Jersey, 2005, vol. 125.

86 R. Ivan, C. Popescu, Á. Pérez del Pino, C. Logofatu and E. György, Appl. Surf. Sci., 2020, 509, 145359.

87 H. Jin, J. Li, Y. Yuan, J. Wang, J. Lu and S. Wang, Adv. Energy Mater., 2018, 8, 1-12.

88 F. Bardé, P. L. Taberna, J. M. Tarascon and M. R. Palacín, J. Power Sources, 2008, 179, 830-836.

89 V. F. Lvovich, Impedance Spectroscopy: Applications to Electrochemical and Dielectric Phenomena, John Wiley \& Sons, 2012.

90 D. Pech, M. Brunet, H. Durou, P. Huang, V. Mochalin, Y. Gogotsi, P. L. Taberna and P. Simon, Nat. Nanotechnol., 2010, 5, 651-654.

91 M. F. El-Kady and R. B. Kaner, Nat. Commun., 2013, 4, 1-9.

92 A. Queraltó, A. P. del Pino, C. Logofatu, A. Datcu, R. Amade, E. Bertran-Serra and E. György, Ceram. Int., 2018, 44, 2040920416.

93 J. Chmiola, P. L. T. Celine Largeot, P. Simon and Y. Gogotsi, Science, 2010, 328, 480-483. 